\title{
Optimal Placement of UAV-Assisted Amplify-and-Forward Relays for Mitigation of Pointing Error in Optical Wireless Backhaul
}

This paper was downloaded from TechRxiv (https://www.techrxiv.org).

\section{LICENSE}

CC BY 4.0

SUBMISSION DATE / POSTED DATE

01-09-2021 / 02-09-2021

\section{CITATION}

Bashir, Muhammad Salman; Alouini, Mohamed-Slim (2021): Optimal Placement of UAV-Assisted Amplifyand-Forward Relays for Mitigation of Pointing Error in Optical Wireless Backhaul. TechRxiv. Preprint. https://doi.org/10.36227/techrxiv.16550916.v1

DOI 


\title{
Optimal Placement of UAV-Assisted Amplify-and-Forward Relays for Mitigation of Pointing Error in Optical
}

\section{Wireless Backhaul}

Muhammad Salman Bashir, Senior Member, IEEE, and Mohamed-Slim Alouini, Fellow, IEEE

\begin{abstract}
The angular instability of unmanned-aerial-vehicle (UAV) relays as well as the turbulence-induced beam wander may lead to significant pointing error in free-space optical backhaul. The hop distance between each relay in a serial relay scheme is an important parameter that can be optimized in order to enhance the performance of the system. In this study, we have optimized the error and outage probabilities as a function of the hop distance vector for amplify-and-forward relays (AF) under a number of constraints such as the maximum and average relay power and the maximum relay gain. We have discovered that for the unconstrained optimization problem, the optimum hop distances is a strictly increasing sequence for both the error and outage probabilities. For maximum power constraint optimization, the optimum hop distances form a strictly increasing sequence, whereas for maximum gain constraint optimization, the optimum hop distances lead to a nondecreasing sequence.
\end{abstract}

\section{Index Terms}

Amplify-and-forward relays, angle-of-arrival, free-space optics, hop distance, pointing error, UAV relays

This work was supported by the Office of Sponsored Research (OSR) at King Abdullah University of Science and Technology (KAUST). (Corresponding author: Muhammad Salman Bashir.) The authors are with the Computer, Electrical and Mathematical Science and Engineering (CEMSE) Division, King Abdullah University of Science and Technology, Thuwal 23955-6900, Saudi Arabia (e-mail: muhammad.bashir@ fulbrightmail.org, slim.alouini@kaust.edu.sa). 


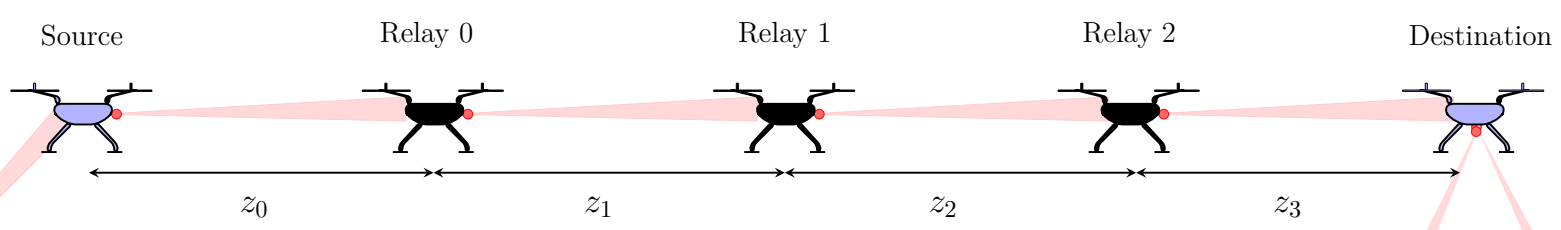

$\underline{\underline{\alpha}}$

Laser Transmitter at Core Network

\section{UAV Backhaul}

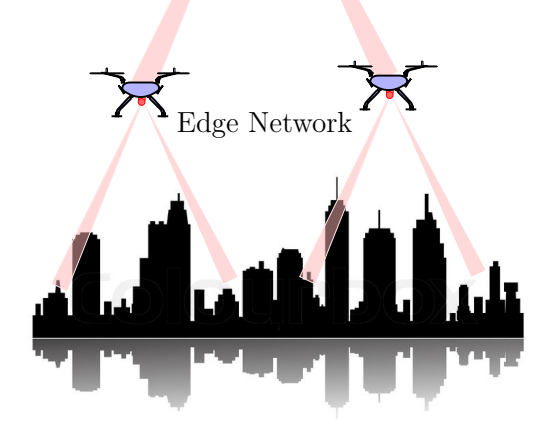

Fig. 1. This figure depicts a network of five hovering UAV relays in wireless backhaul. The locations of the source and the destination relay (marked in blue) are fixed. However, the positions of other relays (marked in black) can be varied in order to minimize the error and outage probabilities of the backhaul link. The number of hops in the backhaul is $M=4$.

\section{INTRODUCTION}

Free-space optical (FSO) communication will play an important role in 6G wireless backhaul where a number of UAV relays will be interconnected through optical links [1]. Such an optically connected wireless backhaul is necessary in order to provide connectivity to remote geographic locations where it is difficult to reach through a conventional communications infrastructure (such as fiber optic cables). The wireless backhaul consists of either a serial configuration of a number of relays, or a parallel configuration, or a mixture of both. Fig. 1 illustrates an example of a serial relay scheme in the backhaul where five relays are used to close the backhaul link.

One of the major issues that limit the performance of free-space optical links in the backhaul is the presence of pointing error [2], [3]. The pointing error is the error caused by the random fluctuation in the angle-of-arrival at the receiver that leads to the incoming beam and the receiver aperture pointing in different directions which leads to a loss in energy captured by the receiver. The effect of the pointing error in free-space optics can be significant since the beam divergence in the optical domain of electromagnetic spectrum is very small compared to microwave frequencies. Therefore, the slightest (angular) vibrations in the UAV relays can cause significant loss of energy at the receiver [4]. There are a number of probability distributions with which the pointing error at the receiver can be modeled: i) Rayleigh, if the error in 


\begin{tabular}{c|c}
\hline$\theta$ & beamwidth (radians) \\
$\phi$ & angle-of-arrival at receiver \\
$\gamma$ & angle-of-arrival variance to beamwidth squared ratio $\frac{\sigma^{2}}{\theta^{2}}$ \\
$\alpha$ & attenuation coefficient due to fog/dirt \\
$\sigma^{2}$ & angle-of-arrival variance \\
$P_{t}$ & transmitted power by first relay \\
$M$ & total number of hops in backhaul \\
$z_{m}$ & hop distance of $m$ th hop $0 \leq m \leq M-1$ \\
$\boldsymbol{z}$ & hop distance vector \\
$\mathcal{P}_{u}$ & power rating (maximum power) of a relay \\
$\mathcal{G}_{u}$ & gain rating (maximum gain) of a relay \\
$a$ & aperture radius \\
$D$ & total backhaul link distance \\
$\sigma_{n}^{2}$ & receiver thermal noise variance \\
\hline & TABLE I \\
& List of SymBoLs
\end{tabular}

each of the two dimensions are zero-mean i.i.d Gaussian random variables, and ii) the Beckmann model which is a more general model than Rayleigh distribution that caters to nonzero-mean errors with unequal variance in the two dimensions [5]. For more literature on generalized pointing error models, the reader is referred to [6], [7], [8].

Even though highly stable UAVs have been developed for 6G wireless connectivity whose angular stability is on the order of a few milliradians [9], it may still be not enough to minimize the effect of pointing loss if the beamwidth of the optical signal is chosen to be small in order to acheive a longer propagation distance. To give an example, the pointing error at a distance $D$ from the transmitter is about $D \phi$ for small $\phi$ approximation where $\phi$ is the angular deviation or the nonzero angle-of-arrival. We term the quantity $D \phi$ as the pointing error which depends on the angle-of-arrival $\phi$ and the distance $z$. For a distance $D=1000 \mathrm{~m}$ and $\phi=1 \mathrm{mrad}$, a pointing error of $1 \mathrm{~m}$ is experienced at the receiver. If a beamwidth of $\theta=1 \mathrm{mrad}$ is chosen for optical signal transmission, a beam radius of $D \theta=1 \mathrm{~m}$ occurs at the receiver location. In this case, a pointing error of $1 \mathrm{~m}$ will cause a sufficient loss in energy at the receiver.

Most of the current literature on FSO multi-hop relaying considers altering two parameters in order to minimize the effect of pointing error: i) beamwidth and ii) the receiver field-of-view (FOV). However, the distance between each relay in multi-hop relaying scheme is an important parameter which has a significant effect on the pointing error. The optimization (minimization) of error or outage probability as a function of hop distance vector (the vector consisting of hop distances in the backhaul) is attractive from 
the perspective of the flexibility of the hop distance vector. In case the beamwidth and the receive FOV of each relay is fixed and cannot be optimized, the location of each relay along the end-to-end link can still be altered at any time without incurring any cost or modification of hardware. For instance, the hop distance vector $\boldsymbol{z}:=\left[\begin{array}{llll}z_{0} & z_{1} & z_{2} & z_{3}\end{array}\right]^{T}$ in Fig. 1 can be varied to find the optimal hop distance vector that will minimize the end-to-end error and outage probabilities of the backhaul given that the total distance $D:=\sum_{m=0}^{3} z_{m}$ is fixed. In our opinion, this flexibility of the hop distance vector makes it an attractive parameter for the optimization of system performance.

\section{Background Literature Review and Motivation for Current Study}

In order to minimize the effect of pointing error of a hovering UAV or satellite relay in a free space optical link, the parameters that have been given the most consideration are the beamwidth and the receiver field-of-view (FOV). For instance, each of the studies [5], [10], [11], [12], [9] has attempted to mitigate the pointing error loss by optimizing the beamwidth and the receiver FOV. However, for amplify-and-forward (AF) relay scheme, the distance between each relay (UAV relays in terrestrial FSO and satellite relays in deep space communications) forms an important parameter vector over which the pointing error is heavily dependent. Thus, there is a potential to mitigate the pointing error loss by optimizing a certain metric — such as the error or outage probability—over the hop distance 1 vector parameter space. In the presence of angular pointing error, the optimization of the error/outage probabilities as a function of $z$ is a problem that has not been given a consideration in previous studies. For instance, in [13] and [9], the multi-relay scheme considers only the case of uniformly placed arrays (distance between each relay is the same) between the transmitter and receiver.

Having said that, there are a few studies that have considered the optimal hop distance configuration for the case of decode-and-forward (DF) relays. However, they still that do not take into account the effect of pointing error (these studies only consider the turbulence-induced scintillation model). For instance, the studies [14], [15] consider the outage probability optimization of multi-hop relaying for serial and parallel relay configurations for lognormal fading channels. They have shown that-if one is to minimize the outage probability - the optimal serial configuration of relay locations is the uniform or equidistant scheme where the distance of each hop is the same. The study [16] builds on the work [14], [15] and derives the optimal serial relay configuration when obstacles are present in the path between the transmitter and receiver for a lognormal fading channel. Having said that, we mention here that these studies also did

${ }^{1}$ We define the hop distance as the distance between two relays that correspond to a particular hop. 
not take into account important constraints—-such as power and gain constraints of each relay-in their optimization problem formulation. The power constraints exist because of the maximum current carrying capacity and the maximum laser transmitter power at each relay, and the gain constraints exist in order to avoid the nonlinear region of transistor amplifiers which occurs at very high gain values.

\section{A. Contributions of This Paper}

In this study, we have undertaken the optimization of the error probability and outage probability bound of the free-space optical link as a function of hop distance vector $z$ for the AF serial relay scheme. The relay positioning or hop distance optimization for DF relays is straightforward if the angle-of-arrival variance at each hop channel is the same 2 , and therefore, have been left out of this discussion. In this study, we assume the same angle-of-arrival variance for each hop, and the variable angle-of-arrival case is reserved for a future study on this topic.

For the AF relays, the optimization of relay positions is carried out under different constraints: the maximum and average relay power constraints and the maximum relay gain constraint. The constrained optimization problem is solved with the help of Karush-Kuhn-Tucker conditions. Under the condition that the channel attenuation coefficient $\alpha \approx 0$ ( $\alpha$ is defined in (7)), we were able to obtain analytical results for majority of cases. We now state some of the important results that we have obtained in this study:

1) For the unconstrained optimization for both the error and outage probabilities, the optimum hop distance is a strictly increasing sequence $z_{0}<z_{1}<\cdots<z_{M-1}$. The sequence values depend on the factor $\gamma$, where $\gamma$ is the angle-of-arrival variance to beamwidth square ratio. If $\gamma=0$ (no pointing error), the optimal configuration is the equidistant or uniform hop distance scheme where the distance between each relay in the link is equal. This result is shown for a low attenuation factor $\alpha$ of the channel.

2) For optimization under gain constraint, the optimization of error and outage probabilities yields an optimal hop distance $z^{\star}$ whose first $M-2$ values are equal: $z_{0}=z_{1}=\cdots=z_{M-2}<z_{M-1}$. We term the vector of type $z^{\star}$ as a quasiequidistant vector. Also, when $\gamma \approx 0$, the error and outage probability minimization under maximum power constraint yields optimal hop distance vectors which are quasiequidistant. However, when $\gamma>>0$, the optimization of outage probability bound under maximum power constraint has been shown to yield a strictly increasing sequence that depends on $\gamma$

2If channel conditions do not change from one hop to the next, an equidistant positioning scheme is the optimal scheme. 
3) When total average power of the system is constant, a large number of low power relays is more advantageous from the error probability perspective when channel conditions are relatively good (small $\gamma$ ). Conversely, for poor channel conditions (large $\gamma$ ), we want to deploy a small number of high power relays in order to minimize the error probability. With regards to outage probability, only a certain number of relays will minimize the outage probability bound for a fixed total average power.

\section{B. Model Assumptions}

We have made the following set of assumptions in this study:

1) The pointing error is assumed to follow the Rayleigh distribution. This follows from our model in which the (solid) angle-of-arrival fluctuations due to hover at the transmitter side are modeled by Rayleigh distribution. The angle-of-arrival (defined by $\phi$ ) statistics for all the relays are assumed to be same but independent of each other ${ }^{3}$

2) We have assumed that the power and gain constraints on each relay are the same. This is justifiable in a scenario where relays with similar power and gain ratings are deployed to close the link.

3) The energy loss due to the angular fluctuations of the receiver aperture is considered negligible due to high angular stability assumption of UAV relays. In other words, only the angle-of-arrival fluctuations at the transmitter side determine the total pointing loss.

4) We assume that the turbulence-induced scintillation is negligible because of the shorter hop distance between each relay. This assumption is built on the fact that the beam radius is usually small relative to the eddy sizes in the uplink because of which the turbulence-induced beam wander is a more prominent factor as compared to scintillation [17]. Thus, we only consider turbulence-induced beam wander effect in our analysis which is modeled by a Gaussian distribution in each of the two dimensions at the receiver [18]. The pointing error caused by beam wander and the angular vibrations of UAVs are treated independently, and lumped into one pointing error variable which is assumed to be Rayleigh distributed at each hop. Optimization of relay positioning under turbulenceinduced scintillation will be incorporated in the next study on this topic where longer hop distances will be considered.

5) We have assumed a CSI-assisted relay scheme in which the gain at a certain relay is equal to the inverse of the channel gain of the hop [13], [19]. Since the channel gain is a function of the hop

${ }^{3}$ The scale of Rayleigh distribution for angle-of-arrival $\phi$ is the same for each relay. 
distance (the larger the hop distance, the smaller the weight), the gain at each relay a function of the hop distance as well. This helps us to express the objective function in terms of the hop distance parameter wherever possible since the main objective of this study is to highlight the dependence of the system performance on the hop distance of each relay in the link.

We have not discussed the performance of fixed-gain relays in order to save on space in this study. Having said that, the models and derivations of CSI-assisted relays in this study are general enough to cater to an analysis of system performance with fixed-gain relays.

6) We have assumed a Gaussian noise model at the receiver in this paper. The underlying assumption is that the received signal strength is strong enough so that a Gaussian assumption is justified, and not so strong such that the signal-dependent Gaussian noise model holds. This assumption is made in order to simplify the analysis.

7) To simplify the arguments in this paper, we have assumed a point-receiver scenario in which the footprint of the receiver aperture is much smaller than the footprint of the beam at the receiver. This assumption is justified when the distance between the transmitter and the receiver is large enough [20].

\section{Organization of Paper}

This paper is organized as follows. Section III defines the channel model under the pointing error. Section IV considers the error probability derivation and Section $\mathrm{V}$ discusses the optimization of error

probability as a function of hop distance vector under different constraints. Section VI explains the derivation and minimization of outage probability bound expressions. Section VII introduces simulation results, and Section VIII sums up the conclusions of this study.

A list of important symbols used in this study is shown in Table $\mathbb{I}$.

\section{Pointing Error Characterization}

For a Gaussian beam, let the received power at a distance $z$ from the transmitter (without any pointing error) be given by

$$
S(z) \approx \frac{2 P_{t}}{2 \pi w^{2}(z)} \exp \left(-\frac{x^{2}+y^{2}}{2 w^{2}(z)}\right) \pi a^{2}=\frac{2 P_{t}}{2 \pi w^{2}(z)} \exp \left(-\frac{r^{2}}{2 w^{2}(z)}\right) \pi a^{2},
$$


where $r$ is radial distance between the center of the circularly symmetric Gaussian beam and the center of the aperture, and $a$ is the (small) aperture radius in meters. The beam radius $w(z)$ at a distance $z$ from the transmitter is expressed as

$$
w(z)=\tan (\theta) z \stackrel{\text { small }}{\approx} \theta z
$$

where $\theta$ is the beamwidth and is given by $\theta \approx \frac{\lambda}{\pi w_{0}}$ for large enough link distance. The quantity $\lambda$ is the wavelength and $w_{0}$ is known as the beam waist. Both these quantities are measured in meters.

Let the pointing error (the Euclidean distance between the center of the beam and the center of the receive aperture) at a distance $z$ from the transmitter be given by

$$
R(z):=\tan (\phi) z \stackrel{\text { small }}{\approx} \phi z
$$

where $\phi$ is the solid angle-of-arrival at the receiver that models the angular instability of the hovering UAV relay as well as the beam wander. We define $\phi:=\sqrt{\phi_{x}^{2}+\phi_{y}^{2}}$ where $\phi_{x}$ is the one-dimensional angle along $x$-axis, and $\phi_{y}$ is the one-dimensional angle along $y$-axis. We model the one-dimensional angles as independent Gaussian random variables with equal variance: $\phi_{x} \sim \mathcal{N}\left(0, \sigma^{2}\right), \phi_{y} \sim \mathcal{N}\left(0, \sigma^{2}\right)$. This implies that $R(z)$ is a Rayleigh random variable whose scale parameter $\sigma_{R}(z):=z \sigma$.

The beamwidth $\theta$ and angle-of-arrival $\phi$ are depicted in Fig. 2.

\section{A. Random Channel Gain}

When the point-detector assumption is true (Assumption 5 in Section II-B, we have that $\pi a^{2}<<\pi w^{2}(z)$ which implies $a<<w(z)$. In this case, the received power in the presence of (random) pointing error at a distance $z$ is given by

$$
S(z) \approx \frac{2 P_{t}}{2 \pi w^{2}(z)} \exp \left(-\frac{R^{2}}{2 w^{2}(z)}\right) \pi a^{2} e^{-\alpha z}
$$

where $2 P_{t}$ is the transmitted power when an OOK pulse is transmitted (average power being $P_{t}$ ), and $R$ is a Rayleigh random variable with scale parameter $\sigma_{R}$. In this case, the random channel gain for a hop link with distance $z$ is defined as

$$
\mathfrak{h}(z):=\frac{1}{2 \pi w^{2}(z)} \exp \left(-\frac{R^{2}}{2 w^{2}(z)}\right) \pi a^{2} e^{-\alpha z}=\frac{a^{2} e^{-\alpha z}}{2 \theta^{2} z^{2}} \exp \left(-\frac{R^{2}}{2 \theta^{2} z^{2}}\right)
$$




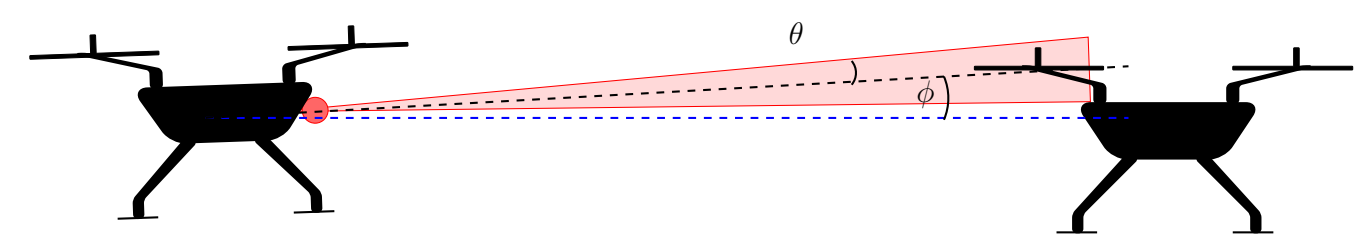

Fig. 2. This figure depicts the non-zero angle-of-arrival $\phi$ and the beamwidth $\theta$. The angle-of-arrival is the angle between the horizontal (line joining the two UAVs in the figure) and the beam axis.

which implies that $S(z)=2 P_{t} \mathfrak{h}(z)$. The average received signal power $\mathcal{S}(z):=\mathbb{E}[S(z)]$ is

$$
\begin{aligned}
\mathcal{S}(z) & \approx \int_{0}^{\infty} \frac{2 P_{t}}{2 \pi w^{2}(z)} \exp \left(-\frac{r^{2}}{2 w^{2}(z)}\right) \pi a^{2} e^{-\alpha z} f_{R(z)}(r) d r \\
& =2 P_{t} \pi a^{2} e^{-\alpha z} \int_{0}^{\infty} \frac{1}{2 \pi w^{2}(z)} \exp \left(-\frac{r^{2}}{2 w^{2}(z)}\right) \frac{r}{\sigma_{R}^{2}(z)} \exp \left(-\frac{r^{2}}{2 \sigma_{R}^{2}(z)}\right) d r \\
& =2 P_{t} \pi a^{2} e^{-\alpha z} \int_{0}^{\infty} \frac{r}{2 \pi w^{2}(z) \sigma_{R}^{2}(z)} \exp \left(-\frac{r^{2}}{2 \frac{w^{2}(z) \sigma_{R}^{2}(z)}{w^{2}(z)+\sigma_{R}^{2}(z)}}\right) d r \\
& =\frac{2 P_{t} \pi a^{2} e^{-\alpha z}}{w^{2}(z)+\sigma_{R}^{2}(z)} \int_{0}^{\infty} \frac{r}{2 \pi\left(\frac{w^{2}(z) \sigma_{R}^{2}(z)}{w^{2}(z)+\sigma_{R}^{2}(z)}\right)} \exp \left(-\frac{r^{2}}{2 \frac{w^{2}(z) \sigma_{R}^{2}(z)}{w^{2}(z)+\sigma_{R}^{2}(z)}}\right) d r \\
& =\frac{2 P_{t} \pi a^{2} e^{-\alpha z}}{2 \pi\left(w^{2}(z)+\sigma_{R}^{2}(z)\right)}=2 P_{t}\left(\frac{\pi a^{2} e^{-\alpha z}}{2 \pi z^{2}\left(\theta^{2}+\sigma^{2}\right)}\right)=2 P_{t} h(z)
\end{aligned}
$$

where $h(z)$ is the mean channel gain. It is defined as

$$
h(z):=\mathbb{E}[\mathbb{h}(z)]=\frac{\pi a^{2}}{2 \pi z^{2}\left(\theta^{2}+\sigma^{2}\right)} e^{-\alpha z}=\frac{a^{2}}{2 z^{2}\left(\theta^{2}+\sigma^{2}\right)} e^{-\alpha z}=\frac{a^{2}}{2 z^{2} \theta^{2}(1+\gamma)} e^{-\alpha z} .
$$

The mean channel gain depends on the variance of angle-of-arrival $\sigma^{2}$, the beamwidth $\theta$, the receiver aperture radius $a$, and the attenuation coefficient $\alpha$. The factor $\gamma$ is the angle-of-arrival variance to beamwidth square ratio: $\gamma:=\frac{\sigma^{2}}{\theta^{2}}$. Additionally, it can be shown that

$$
\mathbb{E}\left[\mathbb{h}^{2}(z)\right]=\frac{a^{4} e^{-2 \alpha z}}{4 \theta^{2} z^{4}\left(\theta^{2}+2 \sigma^{2}\right)}=\frac{a^{4} e^{-2 \alpha z}}{4 \theta^{4} z^{4}(1+2 \gamma)}
$$

\section{Error Probability}

Let us denote the $m$ th hop distance (distance between $(m-1)$ th and $m$ th relay) by $z_{m}$ for $0 \leq m \leq M-1$, where $M$ is the total number of hops. Then, the total (backhaul) link distance $D:=\sum_{m=0}^{M-1} z_{m}$. The instantaneous received signal plus noise (during OOK pulse time) at the first relay (Relay 0) in the 
backhaul is given by

$$
Y_{0}=2 P_{t} \mathfrak{h}\left(z_{0}\right)+N_{0}
$$

where $N_{0}$ represents Gaussian noise at the receiver: $N_{0} \sim \mathcal{N}\left(0, \sigma_{n}^{2}\right)$.

By using the definition of AF relays, Relay 0 multiplies the received signal by a constant gain $G_{0}$, and then retransmits the resulting signal to the second relay in the serial link. The instantaneous received signal plus noise at the second relay (Relay 1) is

$$
Y_{1}=\left(2 P_{t} \mathfrak{h}\left(z_{0}\right)+N_{0}\right) G_{0} \mathfrak{h}\left(z_{1}\right)+N_{1}=2 P_{t} \mathfrak{h}\left(z_{0}\right) \mathfrak{h}\left(z_{1}\right) G_{0}+N_{0} G_{0} \mathfrak{h}\left(z_{1}\right)+N_{1}
$$

where $N_{1} \sim \mathcal{N}\left(0, \sigma_{n}^{2}\right)$. This signal is multiplied by gain $G_{1}$ at the second relay, and transmitted to the third relay and so on.

The instantaneous received signal plus noise at the $m$ th relay-for $0 \leq m \leq M-2$-is

$$
Y_{m}=2 P_{t} \prod_{i=0}^{m} \mathfrak{h}\left(z_{i}\right) \prod_{j=0}^{m-1} G_{j}+N_{0} \prod_{i=1}^{m} \mathfrak{h}\left(z_{i}\right) \prod_{j=0}^{m-1} G_{j}+N_{1} \prod_{i=2}^{m} \mathfrak{h}\left(z_{i}\right) \prod_{j=1}^{m-1} G_{j}+\cdots+N_{m-1} \mathfrak{h}\left(z_{m}\right) G_{m-1}+N_{m}
$$

It can be easily shown that for $M$ hops, the instantaneous received signal plus noise at the destination is $Y_{M-1}=2 P_{t} \prod_{i=0}^{M-1} \mathbb{h}\left(z_{i}\right) \prod_{j=0}^{M-2} G_{j}+N_{0} \prod_{i=1}^{M-1} \mathfrak{h}\left(z_{i}\right) \prod_{j=0}^{M-2} G_{j}+N_{1} \prod_{i=2}^{M-1} \mathfrak{h}\left(z_{i}\right) \prod_{j=1}^{M-2} G_{j}+\cdots+N_{M-2} \mathfrak{h}\left(z_{M-1}\right) G_{M-2}+N_{M-1}$

where we assume that $N_{i}$, for $0 \leq i \leq M-1$, are zero-mean i.i.d. Gaussian random variables with variance $\sigma_{n}^{2}$. In this case, the (backhaul) end-to-end signal and noise variables are given by

$$
\begin{aligned}
& \mathcal{S}:=2 P_{t}\left(\prod_{i=0}^{M-1} \mathfrak{h}\left(z_{i}\right)\right)\left(\prod_{j=0}^{M-2} G_{j}\right) \\
& N:=N_{0}\left(\prod_{i=1}^{M-1} \mathfrak{h}\left(z_{i}\right)\right)\left(\prod_{j=0}^{M-2} G_{j}\right)+N_{1}\left(\prod_{i=2}^{M-1} \mathfrak{h}\left(z_{i}\right)\right)\left(\prod_{j=1}^{M-2} G_{j}\right)+\cdots+N_{M-2} \mathfrak{h}\left(z_{M-1}\right) G_{M-2}+N_{M-1},
\end{aligned}
$$

where $N$ is a zero-mean Gaussian random variable.

Let $P_{m}$ be the instantaneous power delivered by the $m$ th relay. Then, the average transmitted power for 
the $m$ th relay $(0 \leq m \leq M-2)$ is

$$
\bar{P}_{m}=2 P_{t}\left(\prod_{i=0}^{m} h\left(z_{i}\right)\right)\left(\prod_{j=0}^{m} G_{j}\right)
$$

Additionally, from (13) and (14), the instantaneous power delivered by the $m$ th relay

$$
P_{m}=2 P_{t} \prod_{i=0}^{m} \mathfrak{h}\left(z_{i}\right) \prod_{j=0}^{m} G_{j}+\left(N_{0} \prod_{i=1}^{m} \mathfrak{h}\left(z_{i}\right) \prod_{j=0}^{m} G_{j}+N_{1} \prod_{i=2}^{m} \mathfrak{h}\left(z_{i}\right) \prod_{j=1}^{m} G_{j}+\cdots+N_{m} G_{m}\right) .
$$

\section{A. CSI-Assisted Relays}

Here, as discussed in the section on model assumptions (Section II-B), we consider a special case where the gain $G_{m}$ 's are chosen to be the inverse of the mean channel gain: $G_{m}=\frac{1}{h\left(z_{m}\right)}$ for $m=0,1, \ldots, M-2$. Thus, we have that the average received signal power at the destination is

$$
\mu_{\mathcal{S}}:=2 P_{t} h\left(z_{M-1}\right)=\frac{P_{t} a^{2}}{z_{M-1}^{2} \theta^{2}(1+\gamma)} e^{-\alpha z_{M-1}},
$$

and the noise power is

$$
\begin{aligned}
\sigma_{N}^{2} & =\sigma_{n}^{2} e^{-2 \alpha z_{M-1}}\left(\frac{z_{0}^{4}}{z_{M-1}^{4}} e^{2 \alpha z_{0}}\left(\frac{(1+\gamma)^{2}}{1+2 \gamma}\right)^{M-1}+\frac{z_{1}^{4}}{z_{M-1}^{4}} e^{2 \alpha z_{1}}\left(\frac{(1+\gamma)^{2}}{1+2 \gamma}\right)^{M-2}\right. \\
& \left.+\cdots+\frac{z_{M-2}^{4}}{z_{M-1}^{4}} e^{2 \alpha z_{M-2}}\left(\frac{(1+\gamma)^{2}}{1+2 \gamma}\right)+e^{2 \alpha z_{M-1}}\right) \\
& =\sigma_{n}^{2} \frac{e^{-2 \alpha z_{M-1}}}{z_{M-1}^{4}} \sum_{m=0}^{M-1} z_{m}^{4} e^{2 \alpha z_{m}}\left(\frac{(1+\gamma)^{2}}{1+2 \gamma}\right)^{M-(m+1)}
\end{aligned}
$$

where we have assumed that $N_{i}$ are i.i.d. with variance $\sigma_{n}^{2}$, and $N \sim\left(0, \sigma_{N}^{2}\right)$.

In this case, the average transmitted power by the $m$ th relay is

$$
\bar{P}_{m}=2 P_{t}
$$

In order to find the maximum instantaneous transmitted power by the $m$ th relay, we first note that the channel gain $\mathbb{h}$ varies much slower compared to the additive white noise process. In this case, the variance of noise component of $P_{m}$ in $(16)$ can be shown to be

$$
\operatorname{Var}\left[P_{m}\right]=\sigma_{n}^{2}\left(\prod_{i=1}^{m} \mathbb{h}^{2}\left(z_{i}\right) \prod_{j=0}^{m} G_{j}^{2}+\prod_{i=2}^{m} \mathbb{h}^{2}\left(z_{i}\right) \prod_{j=1}^{m} G_{j}^{2}+\cdots+G_{m}^{2}\right)
$$

The maximum value of $\mathrm{h}\left(z_{i}\right)$ is $\frac{a^{2} e^{-\alpha z}}{2 \theta^{2} z^{2}}$. For this case, an upper limit on the variance-after a number of 
steps-can be shown to be

$$
\max \operatorname{Var}\left[P_{m}\right]=\frac{4 \theta^{4} \sigma_{n}^{2}}{a^{4}} \sum_{i=0}^{m} z_{i}^{4} e^{2 \alpha z_{i}}(1+\gamma)^{2(m+1-i)}
$$

and the maximum value of standard deviation is $\frac{2 \theta^{2} \sigma_{n}}{a^{2}} \sqrt{\sum_{i=0}^{m} z_{i}^{4} e^{2 \alpha z_{i}}(1+\gamma)^{2(m+1-i)}}$. We now invoke the assumption that for a zero-mean Gaussian random variable $X, P\left(\left\{X>3 \sigma_{X}\right\}\right) \approx 0$ where $\sigma_{X}$ is the standard deviation of $X$. Using this fact and $(16)$, we obtain the maximum value of $P_{m}$ as

$$
\max P_{m}=2 P_{t}(1+\gamma)^{m+1}+\frac{2 \theta^{2} N^{\star}}{a^{2}} \sqrt{\sum_{i=0}^{m} z_{i}^{4} e^{2 \alpha z_{i}}(1+\gamma)^{2(m+1-i)}}
$$

where $N^{\star}:=3 \sigma_{n}$.

\section{1) Average Bit Error Rate}

The average bit error rate for an OOK symbol is $\mathbb{P}(\mathcal{E})=Q\left(\frac{\mu_{\mathcal{S}}}{2 \sigma_{N}}\right)$, where $\mu_{\mathcal{S}}$ is furnished by (17), $\sigma_{N}$ is provided by the square root of (18), and the function $Q(x):=\frac{1}{\sqrt{2 \pi}} \int_{x}^{\infty} \exp \left(-\frac{u^{2}}{2}\right) d u$. If we set the OOK threshold $\Upsilon_{\text {th }}$ at the point $\mu_{\mathcal{S}} / 2$, the end-to-end probability of correct decision for an on-off keying (OOK) symbol-given that a 0 is transmitted — is given by

$$
\mathbb{P}(\mathcal{C} \mid 0)=P\left(\left\{Y_{M} \leq \Upsilon_{\text {th }}\right\}\right)=P\left(\left\{Y_{M} \leq \frac{\mu_{\mathcal{S}}}{2}\right\}\right)=1-Q\left(\frac{\mu_{\mathcal{S}}}{2 \sigma_{N}}\right)
$$

and, for an equiprobable scheme, it follows that the average bit error rate is

$$
\mathbb{P}(\mathcal{E})=\mathbb{P}(\mathcal{E} \mid 0)=1-\mathbb{P}(\mathcal{C} \mid 0)=Q\left(\frac{\mu_{\mathcal{S}}}{2 \sigma_{N}}\right)
$$

\section{2) Average Total Power Constraint}

It can be shown that the total average transmitted power during OOK pulse time-defined by $P_{a}:=$ $\sum_{m=0}^{M-2} \bar{P}_{m}$-is

$$
P_{a}=(M-1) 2 P_{t}
$$

for the unconstrained gain case. The number $M-1$ is the total number of relays. When there is an upper constraint on the gain, we have (from (19)) that $\bar{P}_{m} \leq 2 P_{t}, \forall m$, and therefore,

$$
P_{a} \leq(M-1) 2 P_{t}
$$


Thus, for some upper limit $\mathcal{P}_{a}$ on the total average power, if we set $P_{t}=\frac{\mathcal{P}_{a}}{2(M-1)}$, then the total average power $P_{a} \leq \mathcal{P}_{a}$ for all possible values of $z_{m}$.

\section{3) Maximum Gain Constraint}

Let the maximum gain rating of each relay be $\mathcal{G}_{u}$. For a fixed total average power $\mathcal{P}_{a}$, the gain constraint can be realized as

$$
G_{m}=\frac{1}{h\left(z_{m}\right)}=\frac{2 z_{m}^{2}\left(\theta^{2}+\sigma^{2}\right) e^{\alpha z_{m}}}{a^{2}} \leq \mathcal{G}_{u} \Longrightarrow z_{m}^{2} e^{\alpha z_{m}} \leq \frac{\mathcal{G}_{u} a^{2}}{2 \theta^{2}(1+\gamma)}
$$

for $\quad 0 \leq m \leq M-2$.

\section{4) Maximum Power Constraint}

The maximum transmitted power by any (last) relay is

$$
\max _{m} \max P_{m}=\max P_{M-2}=2 P_{t}(1+\gamma)^{M-1}+\frac{2 \theta^{2} N^{\star}}{a^{2}} \sqrt{\sum_{i=0}^{M-2} z_{i}^{4} e^{2 \alpha z_{i}}(1+\gamma)^{2(M-1-i)}}
$$

Let the maximum power rating of the drone be $\mathcal{P}_{u}$. From (28), the maximum power constraint can be written as

$$
\left(\sum_{m=0}^{M-2} z_{m}^{4} e^{2 \alpha z_{m}}(1+\gamma)^{2(M-(m+1))}\right)^{\frac{1}{2}} \leq \frac{a^{2}\left(\mathcal{P}_{u}-2 P_{t}(1+\gamma)^{M-1}\right)}{2 \theta^{2} N^{\star}}
$$

\section{Optimization of Relay Positions}

In this section, we will minimize the error probability by maximizing the factor

$$
\frac{\mu_{\mathcal{S}}}{2 \sigma_{n}}=\frac{P_{t} a^{2}}{2 \theta^{2}(1+\gamma) \sigma_{n} \sqrt{\sum_{m=0}^{M-1} z_{m}^{4} e^{2 \alpha z_{m}}\left(\frac{(1+\gamma)^{2}}{1+2 \gamma}\right)^{M-(m+1)}}} .
$$

In this regard, we look at two scenarios: i) the factor $\gamma \approx 0$ and $\gamma \neq 0$.

A. $\gamma \approx 0$ case

\section{1) Unconstrained Optimization}

For the unconstrained optimization problem $\left(\mathcal{G}_{u}=\infty, \mathcal{P}_{u}=\infty\right)$, the goal is to maximize the factor

$$
\frac{\mu_{\mathcal{S}}}{2 \sigma_{n}}=\frac{P_{t} a^{2}}{2 \theta^{2}(1+\gamma) \sigma_{n} \sqrt{\sum_{m=0}^{M-1} z_{m}^{4} e^{2 \alpha z_{m}}}}
$$

as a function of $z_{m}$ 's in order to minimize the probability of error. Thus, in order to minimize the probability of error, the quantity in the square root in $(31)-\sum_{m=0}^{M-1} z_{m}^{4} e^{2 \alpha z_{m}}-$ should be minimized. In 
order to simplify the analysis, let us define the normalized distance $x_{m}:=\frac{D}{z_{m}}$ where $0 \leq x_{m} \leq 1$ and $\sum_{m=0}^{M-1} x_{m}=1$. Additionally, let us define the vector $\boldsymbol{x}:=\left[\begin{array}{llll}x_{0} & x_{1} & \cdots & x_{M-1}\end{array}\right]^{T}$. Thus, in order to minimize the probability of error, the goal is to solve the following optimization problem:

$$
\begin{array}{ll}
\underset{x_{0}, \ldots, x_{M-1}}{\operatorname{minimize}} & \sum_{m=0}^{M-1}\left(x_{m} D\right)^{4} e^{2 \alpha x_{m} D} \\
\text { subject to } & \text { i) } \sum_{m=0}^{M-1} x_{m}=1, \\
& \text { ii) } x_{m} \geq 0, \quad 0 \leq m \leq M-1 .
\end{array}
$$

This problem may be solved with only one equality constraint (Constraint 1) and by choosing the positive values of the solution vector $\boldsymbol{x}^{*}$. As can be seen, the objective function is a convex function of the argument $\boldsymbol{x}$, and both constraints also form convex sets. This convex optimization problem may be solved by method of Lagrange multipliers. We define

$$
\mathcal{L}(\boldsymbol{x}, \lambda)=\sum_{m=0}^{M-1}\left(x_{m} D\right)^{4} e^{2 \alpha x_{m} D}-\lambda\left(\sum_{m=0}^{M-1} x_{m}-1\right) .
$$

By calculating the gradient $\nabla_{\boldsymbol{x}, \lambda} \mathcal{L}(\boldsymbol{x}, \lambda)$ and setting it equal to zero, we arrive at the following $M+1$ equations which are also known as the first order Karush-Kuhn-Tucker conditions:

$$
\nabla_{\boldsymbol{x}, \lambda} \mathcal{L}(\boldsymbol{x}, \lambda)=\left[\begin{array}{c}
4 x_{0}^{3} D^{4} e^{2 \alpha x_{0} D}+\left(x_{0} D\right)^{4} e^{2 \alpha x_{0} D} 2 \alpha D-\lambda \\
4 x_{1}^{3} D^{4} e^{2 \alpha x_{1} D}+\left(x_{1} D\right)^{4} e^{2 \alpha x_{1} D} 2 \alpha D-\lambda \\
\vdots \\
4 x_{M-1}^{3} D^{4} e^{2 \alpha x_{M-1} D}+\left(x_{M-1} D\right)^{4} e^{2 \alpha x_{M-1} D} 2 \alpha D-\lambda \\
\sum_{m=0}^{M-1} x_{m}-1
\end{array}\right]=\left[\begin{array}{c}
0 \\
0 \\
\vdots \\
0 \\
0
\end{array}\right]
$$

In the set of equations written above, the first $M$ equations dictate that $x_{0}=x_{1}=\cdots=x_{M-1}$. This result, in addition to the last equation, implies that $x_{m}^{*}=\frac{1}{M}$ for all $m$ is one candidate solution. In order to see if $\boldsymbol{x}^{*}:=\left[\begin{array}{llll}x_{0}^{*} & x_{1}^{*} & \cdots & x_{M-1}^{*}\end{array}\right]$ is a strict local minimizer, we need to know whether the sufficient condition holds, i.e. the Hessian $\nabla_{\boldsymbol{x}}^{2} \mathcal{L}\left(\boldsymbol{x}^{*}, \lambda^{*}\right)$ is a positive definite matrix. It can be easily shown that $\nabla_{\boldsymbol{x}}^{2} \mathcal{L}\left(\boldsymbol{x}^{*}, \lambda^{*}\right)=C \mathbf{I}_{M}$ where $C:=\frac{4 \exp \left(2 \alpha \frac{D}{M}\right) D^{4}}{M^{2}}\left[\frac{\alpha^{2} D^{2}}{M^{2}}+\frac{4 \alpha D}{M}+3\right]$, and $\mathbf{I}_{M}$ is an $M \times M$ identity matrix. Since $C>0$, we have that the Hessian is a positive definite matrix, and that $\boldsymbol{x}^{*}$ is a strict local minimizer of the probability of error.

This concludes the following result: In order to minimize the probability of error for the unconstrained case, the relays should be placed at equidistant points along the backhaul link. 


\section{2) Constrained Optimization}

In order to minimize the probability of error under the maximum gain constraint, we solve the following optimization problem:

$$
\begin{array}{ll}
\underset{z_{0}, \ldots, z_{M-1}}{\operatorname{minimize}} & \sum_{m=0}^{M-1}\left(x_{m} D\right)^{4} e^{2 \alpha x_{m} D} \\
\text { subject to } & \text { i) } \sum_{m=0}^{M-1} x_{m}=1, \\
& \text { ii) } x_{m} \geq 0, \quad 0 \leq m \leq M-1, \\
& \text { iii) } x_{m}^{2} e^{\alpha x_{m} D} \leq \frac{\mathcal{G}_{u} a^{2}}{2 D^{2} \theta^{2}}, \quad 0 \leq m \leq M-2 .
\end{array}
$$

We note that if $\mathcal{G}_{u} \geq \frac{2 \exp \left(\alpha \frac{D}{M}\right) D^{2} \theta^{2}}{a^{2} M^{2}}$, then the inequality constraint is inactive and the optimal point is $\boldsymbol{x}^{*}=\left[\begin{array}{llll}\frac{1}{M} & \frac{1}{M} & \cdots & \frac{1}{M}\end{array}\right]^{T}$. Otherwise, the inequality constraint is active, and the optimal point $\boldsymbol{x}^{*}$ resides on the boundary of the constraint. Since there is only one boundary point for the maximum gain constraint-which is given by $x_{m}^{2} e^{\alpha x_{m} D}=\frac{\mathcal{G}_{u} a^{2}}{2 D^{2} \theta^{2}}$ for all $m, 0 \leq m \leq M-2$-we have that the optimal point satisfies $\left(x_{m}^{*}\right)^{2} e^{\alpha x_{m}^{*} D}=\frac{\mathcal{G}_{u} a^{2}}{2 D^{2} \theta^{2}}$ for $0 \leq m \leq M-2$. For $\alpha \approx 0$, the optimal point is

$$
x_{m}^{*}=\sqrt{\frac{\mathcal{G}_{u}}{2}} \frac{a}{D \theta}, \quad 0 \leq m \leq M-2,
$$

and $x_{M-1}^{*}=1-(M-1) \sqrt{\frac{\mathcal{G}_{u}}{2}} \frac{a}{D \theta}$.

\section{3) Constrained Optimization of Relay Positions: Maximum Power Constraint}

In order to minimize the probability of error under the maximum power constraint, we solve the following optimization problem:

$$
\begin{array}{ll}
\underset{z_{0}, \ldots, z_{M-1}}{\operatorname{minimize}} & \sum_{m=0}^{M-1}\left(x_{m} D\right)^{4} e^{2 \alpha x_{m} D} \\
\text { subject to } & \text { i) } \sum_{m=0}^{M-1} x_{m}=1, \\
& \text { ii) } x_{m} \geq 0, \quad 0 \leq m \leq M-1, \\
& \text { iii) }\left(\sum_{m=0}^{M-2} D^{4} x_{m}^{4} e^{2 \alpha x_{m} D}\right)^{\frac{1}{2}} \leq \frac{a^{2}\left(\mathcal{P}_{u}-2 P_{t}\right)}{2 N^{\star} \theta^{2}} .
\end{array}
$$

In this constrained optimization problem, the inequality constraint is inactive when

$$
\mathcal{P}_{u} \geq \frac{\sqrt{M-1}}{M^{2}} e^{\alpha \frac{D}{M}} \frac{2 D^{2} N^{\star} \theta^{2}}{a^{2}}+2 P_{t}
$$


and the optimal point is $\boldsymbol{x}^{*}=\left[\begin{array}{cccc}\frac{1}{M} & \frac{1}{M} & \cdots & \frac{1}{M}\end{array}\right]^{T}$ which implies that the optimal point $\boldsymbol{z}^{*}=D \boldsymbol{x}^{*}$. Otherwise, the inequality constraint is active, and the optimal point exists on the boundary of the constraint. The boundary point in this case is the set of all points $\boldsymbol{x}_{B}$ so that the equality $\left(\sum_{m=0}^{M-2} x_{m}^{4} e^{2 \alpha x_{m} D}\right)^{\frac{1}{2}}=$ $\frac{a^{2}\left(\mathcal{P}_{u}-2 P_{t}\right)}{2 D^{2} \sigma_{n}^{\star}\left(\theta^{2}+\sigma^{2}\right)}$ holds. We call such a set containing all boundary points $B$. Additionally, for the purpose of finding the optimal set $\boldsymbol{x}^{*}$ in this case, we say that a $M \times 1$ vector $\boldsymbol{x}$ is quasiequidistant if the first $M-1$ elements of $\boldsymbol{x}$ are equal to each other.

We now show that the optimal optimal hop distance vector in this case is $\boldsymbol{z}_{B}^{*} \in B$ is a quasiequidistant vector given by

$$
\tilde{\boldsymbol{z}}_{B}:=\left[\begin{array}{lllll}
\frac{D_{0}}{M-1} & \frac{D_{0}}{M-1} & \cdots & \frac{D_{0}}{M-1} & \left(D-D_{0}\right)
\end{array}\right]^{T}
$$

for some $D_{0}<(M-1) \frac{D}{M}$ for which the constraint equality holds, i.e. $\left(\sum_{m=0}^{M-2}\left(\frac{D_{0}}{M-1}\right)^{4} e^{2 \alpha \frac{D_{0}}{M-1}}\right)^{\frac{1}{2}}=$ $\frac{a^{2}\left(\mathcal{P}_{u}-2 P_{t}\right)}{2 \sigma_{n}^{\star}\left(\theta^{2}+\sigma^{2}\right)}$. This implies that $\tilde{\boldsymbol{z}}_{B}$ lies in $B$ and the sum of its first $M-1$ elements is $D_{0}$ where $D-D_{0}>\frac{D_{0}}{M}$.

a) Proof by Contradiction: Let us assume that there exists a vector

$$
\boldsymbol{z}_{B}^{\prime}:=\left[\begin{array}{lllll}
D_{0}^{\prime} x_{0}^{\prime} & D_{0}^{\prime} x_{1}^{\prime} & \cdots & D_{0}^{\prime} x_{M-2}^{\prime} & D-D_{0}^{\prime}
\end{array}\right]^{T}
$$

such that $\sum_{m=0}^{M-2} x_{m}^{\prime}=1$. This vector lies in $B$, that $\boldsymbol{z}_{B}^{\prime} \neq \tilde{\boldsymbol{z}}_{B}$, and yet minimizes the error probability. Since $\boldsymbol{z}_{B}^{\prime} \in B$, the sum of the first $M-1$ elements of $\boldsymbol{z}_{B}^{\prime}$ must equal $D_{0}^{\prime}$ where $D_{0}^{\prime} \leq D_{0}$. However, we know that another $M \times 1$ quasiequidistant vector

$$
\tilde{\boldsymbol{z}}^{\prime}:=\left[\begin{array}{ccccc}
\frac{D_{0}^{\prime}}{M-1} & \frac{D_{0}^{\prime}}{M-1} & \cdots & \frac{D_{0}^{\prime}}{M-1} & \left(D-D_{0}^{\prime}\right)
\end{array}\right]^{T}
$$

will strictly minimize the term $\left(\sum_{m=0}^{M-2}\left(x_{m} D_{0}^{\prime}\right)^{4} e^{2 \alpha x_{m} D_{o}^{\prime}}\right)^{\frac{1}{2}}$ compared to $\boldsymbol{z}_{B}^{\prime}$. Hence, this point satisfies the power constraint (even though the constraint is satisfied with a strict inequality which implies that $\left.\tilde{\boldsymbol{z}}^{\prime} \notin B\right)$. Additionally,

$$
\left(\sum_{m=0}^{M-2}\left(\frac{D_{0}^{\prime}}{M-1}\right)^{4} e^{2 \alpha \frac{D_{0}^{\prime}}{M-1}}\right)+\left(D-D_{0}^{\prime}\right)^{4} e^{2 \alpha\left(D-D_{0}^{\prime}\right)}<\left(\sum_{m=0}^{M-2}\left(x_{m}^{\prime} D_{0}^{\prime}\right)^{4} e^{2 \alpha x_{m}^{\prime} D_{0}^{\prime}}\right)+\left(D-D_{0}^{\prime}\right)^{4} e^{2 \alpha\left(D-D_{0}^{\prime}\right)}
$$

where the quantity on the left of the inequality is the objective function corresponding to $\tilde{\boldsymbol{z}}^{\prime}$, and the quantity on the right of the inequality is the objective function corresponding to $\boldsymbol{z}_{B}^{\prime}$. Thus, $\mathbb{P}\left(\tilde{\boldsymbol{z}}^{\prime}\right) \leq \mathbb{P}\left(\boldsymbol{z}_{B}^{\prime}\right)$. Additionally, $\mathbb{P}\left(\tilde{\boldsymbol{z}}_{B}\right) \leq \mathbb{P}\left(\tilde{\boldsymbol{z}}^{\prime}\right)$ since $\tilde{\boldsymbol{z}}_{B} \in B$ and $\tilde{\boldsymbol{z}}^{\prime} \notin B$. Thus, we have that $\mathbb{P}\left(\tilde{\boldsymbol{z}}_{B}\right) \leq \mathbb{P}\left(\boldsymbol{z}_{B}^{\prime}\right)$, which is a 
contradiction. Hence, it follows that $\boldsymbol{z}_{B}^{*}=\tilde{\boldsymbol{z}}_{B}$.

\section{B. $\gamma>>0$ Case}

\section{1) Unconstrained Optimization}

The unconstrained optimization problem is stated as follows:

$$
\begin{array}{ll}
\underset{x_{0}, \ldots, x_{M-1}}{\operatorname{minimize}} & \sum_{m=0}^{M-1}\left(x_{m} D\right)^{4} e^{2 \alpha x_{m} D}\left(\frac{(1+\gamma)^{2}}{1+2 \gamma}\right)^{M-(m+1)} \\
\text { subject to } & \text { i) } \sum_{m=0}^{M-1} x_{m}=1, \\
& \text { ii) } x_{m} \geq 0, \quad 0 \leq m \leq M-1 .
\end{array}
$$

Here, the objective function in (43) is a convex function of $\boldsymbol{x}$ with constraints that form convex sets. Thus, we first solve the first order Karush-Kuhn-Tucker conditions in order to arrive at a candidate solution of this convex optimization problem. In this regard, we first define the Lagrangian

$$
\mathcal{L}(\boldsymbol{x}, \boldsymbol{\lambda}, \boldsymbol{s})=\sum_{m=0}^{M-1}\left(x_{m} D\right)^{4} e^{2 \alpha x_{m} D}\left(\frac{(1+\gamma)^{2}}{1+2 \gamma}\right)^{M-(m+1)}-\lambda_{0}\left(\sum_{m=0}^{M-1} x_{m}-1\right)-\sum_{m=1}^{M} \lambda_{m}\left(x_{m-1}-s_{m-1}\right),
$$

where $\boldsymbol{\lambda}:=\left[\begin{array}{llll}\lambda_{0} & \lambda_{1} & \cdots & \lambda_{M}\end{array}\right]^{T}$ and $\boldsymbol{s}:=\left[\begin{array}{llll}s_{0} & s_{1} & \cdots & s_{M-1}\end{array}\right]^{T}$, where $s_{m}$ is a slack variable and $s_{m} \geq 0$ for $0 \leq m \leq M-1$. However, in order to simplify the optimization, we solve the optimization problem (78) without the inequalities, and see if the resulting solution satisfies the inequality constraints. Therefore, let us define a simpler Lagrangian function as

$$
\mathcal{L}^{\prime}(\boldsymbol{x}, \lambda):=\sum_{m=0}^{M-1}\left(x_{m} D\right)^{4} e^{2 \alpha x_{m} D}\left(\frac{(1+\gamma)^{2}}{1+2 \gamma}\right)^{M-(m+1)}-\lambda\left(\sum_{m=0}^{M-1} x_{m}-1\right)
$$

The first order conditions are given in matrix form as

$$
\nabla_{\boldsymbol{x}, \lambda} \mathcal{L}^{\prime}(\boldsymbol{x}, \lambda)=\left[\begin{array}{c}
\left(4 x_{0}^{3} D^{4} e^{2 \alpha x_{0} D}+\left(x_{0} D\right)^{4} e^{2 \alpha x_{0} D} 2 \alpha D\right)\left(\frac{(1+\gamma)^{2}}{1+2 \gamma}\right)^{M-1}-\lambda \\
\left(4 x_{1}^{3} D^{4} e^{2 \alpha x_{1} D}+\left(x_{1} D\right)^{4} e^{2 \alpha x_{1} D} 2 \alpha D\right)\left(\frac{(1+\gamma)^{2}}{1+2 \gamma}\right)^{M-2}-\lambda \\
\vdots \\
4 x_{M-1}^{3} D^{4} e^{2 \alpha x_{M-1} D}+\left(x_{M-1} D\right)^{4} e^{2 \alpha x_{M-1} D} 2 \alpha D-\lambda \\
\sum_{m=0}^{M-1} x_{m}-1
\end{array}\right]=\left[\begin{array}{c}
0 \\
0 \\
\vdots \\
0 \\
0
\end{array}\right] .
$$


The solution to the set of equations in (46) is not trivial, and would require numerical techniques to solve. However, when $\alpha \approx 0$, we have, from the first $(M)$ equations, that $x_{1}^{*}=\left(\frac{(1+\gamma)^{2}}{1+2 \gamma}\right)^{\frac{1}{3}} x_{0}^{*}$,

$x_{2}^{*}=\left(\frac{(1+\gamma)^{2}}{1+2 \gamma}\right)^{\frac{2}{3}} x_{0}^{*}, \ldots, x_{M-1}^{*}=\left(\frac{(1+\gamma)^{2}}{1+2 \gamma}\right)^{\frac{(M-1)}{3}} x_{0}^{*}$, and from the last equation in the equation set (46), we have that

$$
x_{m}^{*}=\frac{1-\left(\frac{(1+\gamma)^{2}}{1+2 \gamma}\right)^{\frac{1}{3}}}{1-\left(\frac{(1+\gamma)^{2}}{1+2 \gamma}\right)^{\frac{M}{3}}}\left(\frac{(1+\gamma)^{2}}{1+2 \gamma}\right)^{\frac{m}{3}}, \quad 0 \leq m \leq M-1 .
$$

Since $(1+\gamma)^{2} \geq 1+2 \gamma$, we obtain ascending values of $x_{m}^{*}$ 's for the optimal scenario: $x_{0}^{*}<x_{1}^{*}<\cdots<$ $x_{M-1}^{*}$. Additionally, we note that all $x_{m}^{*}>0$.

It is easy to see that $\nabla_{\boldsymbol{x}}^{2} \mathcal{L}^{\prime}\left(\boldsymbol{x}^{*}, \lambda^{*}\right)$ is an $M \times M$ diagonal matrix, whose $m$ th element-for $0 \leq m \leq$ $M-1$-on the diagonal is $\Lambda_{m}:=12\left(x_{m}^{*}\right)^{2} D^{4}\left(\frac{(1+\gamma)^{2}}{1+2 \gamma}\right)^{M-(m+1)}$ which is strictly greater than zero. Hence $\nabla_{\boldsymbol{x}}^{2} \mathcal{L}^{\prime}\left(\boldsymbol{x}^{*}, \lambda\right)$ is a positive definite matrix which implies $\boldsymbol{x}^{*}$ in 47) is a strict local minimizer.

\section{2) Constrained Optimization}

a) Maximum Gain Constraint:

$$
\begin{array}{ll}
\underset{x_{0}, \ldots, x_{M-1}}{\operatorname{minimize}} & \sum_{m=0}^{M-1}\left(x_{m} D\right)^{4} e^{2 \alpha x_{m} D}(1+\gamma)^{2(M-(m+1))} \\
\text { subject to } & \text { i) } \sum_{m=0}^{M-1} x_{m}=1, \\
& \text { ii) } x_{m} \geq 0, \quad 0 \leq m \leq M-1, \\
& \text { iii) } x_{m}^{2} e^{\alpha x_{m} D} \leq \frac{\mathcal{G}_{u} a^{2}}{2 D^{2} \theta^{2}(1+\gamma)}, \quad 0 \leq m \leq M-2 .
\end{array}
$$

For the gain constraint, there is only one point that lies in $B$, which is the optimal point. This optimal point satisfies $x_{0}^{*}=x_{1}^{*}=\cdots=x_{M-2}^{*}$ and $x_{M-1}^{*}=1-\sum_{m=0}^{M-2} x_{m}^{*}$.

For the case when $\alpha \approx 0$, we note from (77) that the gain constraint is active when $\mathcal{G}_{u} \leq \frac{2 D^{2} \theta^{2}(1+\gamma)}{a^{2}}\left(x_{M-2}^{*}\right)^{2}$, where $x_{M-2}^{*}:=\frac{1-\left(\frac{(1+\gamma)^{2}}{1+2 \gamma}\right)^{\frac{1}{3}}}{1-\left(\frac{(1+\gamma)^{2}}{1+2 \gamma}\right)^{\frac{M}{3}}}\left(\frac{(1+\gamma)^{2}}{1+2 \gamma}\right)^{\frac{m}{3}}$. When the constraint is active, the optimal point is $x_{m}^{*}=\sqrt{\frac{\mathcal{G}_{u} a^{2}}{2 D^{2} \theta^{2}(1+\gamma)}}$ for $0 \leq m \leq M-2$, and $x_{M-1}^{*}=1-(M-1) \sqrt{\frac{\mathcal{G}_{u} a^{2}}{2 D^{2} \theta^{2}(1+\gamma)}}$. 
b) Maximum Power Constraint:

$$
\begin{array}{ll}
\underset{x_{0}, \ldots, x_{M-1}}{\operatorname{minimize}} \quad \sum_{m=0}^{M-1}\left(x_{m} D\right)^{4} e^{2 \alpha x_{m} D}\left(\frac{(1+\gamma)^{2}}{1+2 \gamma}\right)^{M-(m+1)} \\
\text { subject to } \quad \text { i) } \sum_{m=0}^{M-1} x_{m}=1, \\
\text { ii) } x_{m} \geq 0, \quad 0 \leq m \leq M-1, \\
\text { iii) }\left(\sum_{m=0}^{M-2} D^{4} x_{m}^{4} e^{2 \alpha x_{m} D}(1+\gamma)^{2(M-(m+1))}\right)^{\frac{1}{2}} \leq \frac{a^{2}\left(\mathcal{P}_{u}-2 P_{t}(1+\gamma)^{M-1}\right)}{2 \theta^{2} N^{\star}} .
\end{array}
$$

The constraint is active when

$$
\mathcal{P}_{u}<\left(\sum_{m=0}^{M-2} D^{4}\left(x_{m}^{*}\right)^{4} e^{2 \alpha x_{m}^{*} D}(1+\gamma)^{2(M-(m+1))}\right)^{\frac{1}{2}} \frac{2 \theta^{2} N^{\star}}{a^{2}}+2 P_{t}(1+\gamma)^{M-1}
$$

where $x_{m}^{*}$ is furnished by 47 .

It is difficult to obtain the optimal point for the power constraint scenario analytically. We will have to resort to numerical optimization techniques to find the optimal point in this case.

\section{Constrained Optimization: Average Power Constraint}

We now ask ourselves the question: "What is the probability of error performance for AF serial relay scheme as the number of relays $M$ in the backhaul grows under the constraint that the total average power of the system is kept constant?" We consider the asymptotic scenario $M \rightarrow \infty$ for two channel conditions: i) when $\gamma \rightarrow 0$ (favorable channel conditions), and ii) when $\gamma \rightarrow \infty$ (adverse channel conditions). Additionally, in order to keep the analysis simple, we only consider the equidistant scheme: $z_{m}=\frac{D}{M}$.

Before we consider the two special scenarios, we reiterate that the average power constraint is satisfied by setting

$$
P_{t}=\frac{\mathcal{P}_{a}}{2(M-1)}
$$

in (31) which would guarantee that the total average power $P_{a} \leq \mathcal{P}_{a}$ for the system.

$$
\text { 1) } \gamma \rightarrow 0
$$

For the case $\gamma \rightarrow 0$, the SNR factor $\frac{\mu_{\mathcal{S}}}{2 \sigma_{n}}$ in $(30)$ reduces to

$$
\frac{\mu_{\mathcal{S}}}{2 \sigma_{n}}=\frac{P_{t} a^{2}}{2 \theta^{2} \sigma_{n} \sqrt{\sum_{m=0}^{M-1} z_{m}^{4} e^{2 \alpha z_{m}}}}
$$


We now consider the equidistant scheme. Substituting $z_{m}=\frac{D}{M}$ and the value of $P_{t}$ from (51), we have that

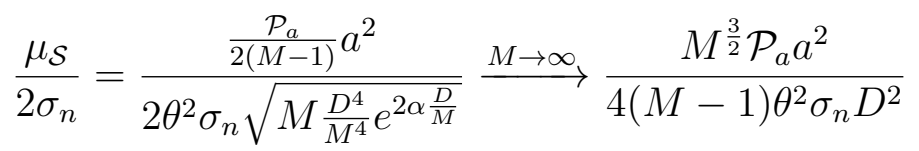

since $\exp \left(2 \alpha \frac{D}{M}\right) \rightarrow 1$ as $M \rightarrow \infty$. We note that-under favorable channel conditions-the SNR factor $\frac{\mu_{\mathcal{S}}}{2 \sigma_{n}}$ in $(53)$ increases with rate $O(\sqrt{M})$ where $O(\cdot)$ stands for Big-O notation.

We state the following fact for the total average power constrained system: When the angle-of-arrival variance is much smaller than the beamwidth square, it is more advantageous to use a large number of low power relays in order to minimize the error probability.

2) $\gamma \rightarrow \infty$

For large $\gamma, \frac{\left(1+\gamma^{2}\right)}{1+2 \gamma} \approx \frac{\gamma}{2}+1$. In this case,

$$
\frac{\mu_{\mathcal{S}}}{2 \sigma_{n}} \approx \frac{P_{t} a^{2}}{2 \theta^{2}(1+\gamma) \sigma_{n} \sqrt{\sum_{m=0}^{M-1} z_{m}^{4} e^{2 \alpha z_{m}}\left(\frac{\gamma}{2}+1\right)^{M-(m+1)}}}
$$

For large $\gamma$, we assume that the terms $\left(\frac{\gamma}{2}+1\right)^{M-2},\left(\frac{\gamma}{2}+1\right)^{M-3}, \ldots,\left(\frac{\gamma}{2}+1\right)$ are much smaller than $\left(\frac{\gamma}{2}+1\right)^{M-1}$ and can be ignored in the denominator. Under this approximation, and substituting the average power constraint from (51), we have that

$$
\frac{\mu_{\mathcal{S}}}{2 \sigma_{n}} \approx \frac{\mathcal{P}_{a} a^{2}}{4(M-1) \theta^{2}(1+\gamma) \sigma_{n} z_{0}^{2} e^{\alpha z_{0}}\left(\frac{\gamma}{2}+1\right)^{\frac{M-1}{2}}}=\frac{M^{2} \mathcal{P}_{a} a^{2}}{4(M-1) \theta^{2}(1+\gamma) \sigma_{n} D^{2} e^{\alpha \frac{D}{M}}\left(\frac{\gamma}{2}+1\right)^{\frac{M-1}{2}}}
$$

which is proportional to $\frac{M}{\left(\frac{\gamma}{2}+1\right)^{\frac{M-1}{2}}}$ for large $M$. Since $\frac{M}{\left(\frac{\gamma}{2}+1\right)^{\frac{M-1}{2}}}$ goes to 0 as $M$ goes to $\infty$, we note that the SNR factor $\frac{\mu_{\mathcal{S}}}{2 \sigma_{n}}$ will decrease monotonically with $M$ for large $M$. This implies that, under adverse channel conditions, the probability of error will grow if more number of relays are introduced in the backhaul.

We state the second fact when the total average power is constrained: When the angle-of-arrival variance is much larger than the beamwidth square, it is important to use a small number of high power relays in order to minimize the error probability. 


\section{End-to-End Outage Probability}

For the AF relay scheme, we say that the end-to-end outage of the backhaul link takes place if the instantaneous SNR at the destination goes below a certain predefined threshold. In this regard, Afer substituting the expression for $\mathfrak{h}\left(z_{i}\right)$, the signal at the destination in the backhaul is given by

$$
\begin{aligned}
Y_{M-1} & =2 P_{t} \frac{\exp \left(-\sum_{i=0}^{M-1} \frac{R_{i}^{2}}{2 w^{2}\left(z_{i}\right)}\right)}{\prod_{j=0}^{M-1}\left(2 \pi w^{2}\left(z_{j}\right)\right)}\left(\pi a^{2}\right)^{M}\left(\prod_{k=0}^{M-2} G_{k}\right)+N_{0}\left(\prod_{k=0}^{M-2} G_{k}\right) \frac{\exp \left(-\sum_{i=1}^{M-1} \frac{R_{i}^{2}}{2 w^{2}\left(z_{i}\right)}\right)}{\prod_{j=1}^{M-1}\left(2 \pi w^{2}\left(z_{j}\right)\right)}\left(\pi a^{2}\right)^{M-1} \\
& +N_{1}\left(\prod_{k=1}^{M-2} G_{k}\right) \frac{\exp \left(-\sum_{i=2}^{M-1} \frac{R_{i}^{2}}{2 w^{2}\left(z_{i}\right)}\right)}{\prod_{j=2}^{M-1}\left(2 \pi w^{2}\left(z_{j}\right)\right)}\left(\pi a^{2}\right)^{M-2} \\
& +\cdots+N_{M-2}\left(\prod_{k=M-2}^{M-2} G_{k}\right) \frac{\exp \left(-\sum_{i=M-1}^{M-1} \frac{R_{i}^{2}}{2 w^{2}\left(z_{i}\right)}\right)}{\prod_{j=M-1}^{M-1}\left(2 \pi w^{2}\left(z_{j}\right)\right)}\left(\pi a^{2}\right)^{M-(M-1)}+N_{M-1}
\end{aligned}
$$

where the signal component in $(56)$ is

$$
\mathcal{S}=\frac{P_{t} a^{2 M} \prod_{k=0}^{M-2} G_{k}}{2^{M-1} \prod_{j=0}^{M-1} w^{2}\left(z_{j}\right)} \times \exp \left(-\left(\sum_{i=0}^{M-1} \frac{R_{i}^{2}}{2 w^{2}\left(z_{i}\right)}+\alpha \sum_{i=0}^{M-1} z_{i}\right)\right)
$$

and the noise component in 56 is $N=\sum_{\ell=0}^{M-1} N_{\ell} \beta_{\ell}$ where

$$
\beta_{\ell}:=\frac{a^{2(M-\ell-1)}\left(\prod_{k=\ell}^{M-2} G_{k}\right)}{2^{M-\ell-1} \prod_{j=\ell+1}^{M-1} w^{2}\left(z_{j}\right)} \exp \left(-\left(\sum_{i=\ell+1}^{M-1} \frac{R_{i}^{2}}{2 w^{2}\left(z_{i}\right)}+\alpha \sum_{i=\ell+1}^{M-1} z_{i}\right)\right), \quad 0 \leq \ell \leq M-2
$$

and $\beta_{M-1}=1$. In this case, the outage probability (conditioned on $\beta_{0}, \ldots, \beta_{M-1}$ ) is given by

$$
\mathbb{P}(\mathcal{O})=\mathbb{P}\left(\left\{\frac{\mathcal{S}^{2}}{\sigma_{n}^{2} \sum_{\ell=0}^{M-1} \beta_{\ell}^{2}} \leq \Upsilon_{\text {th }}\right\}\right)
$$

a) CSI-Assisted Relays: For $G_{i}=\frac{1}{h\left(z_{i}\right)}$, we have that

$$
\begin{aligned}
& \mathcal{S}^{2}=P_{t}^{2}\left(\frac{a}{\theta z_{M-1}}\right)^{4}(1+\gamma)^{2(M-1)} \exp \left(-2 \alpha z_{M-1}\right) \exp \left(-\sum_{i=0}^{M-1} \frac{R_{i}^{2}}{w^{2}\left(z_{i}\right)}\right), \\
& \beta_{\ell}^{2}=\frac{z_{\ell}^{4}}{z_{M-1}^{4}} \exp \left(2 \alpha\left(z_{\ell}-z_{M-1}\right)\right)(1+\gamma)^{2(M-(\ell+1))} \exp \left(-\sum_{i=\ell+1}^{M-1} \frac{R_{i}^{2}}{w^{2}\left(z_{i}\right)}\right) .
\end{aligned}
$$

Note: The quantities $R_{i}^{2}$ are independent exponential random variables with mean $2 \sigma_{R_{i}}^{2}$ for all $i$. Additionally, $\frac{R_{i}^{2}}{w^{2}\left(z_{i}\right)}$ is also exponentially distributed with mean $\frac{2 \sigma_{R_{i}}^{2}}{w^{2}\left(z_{i}\right)}$ or parameter $\lambda_{i}:=\frac{w^{2}\left(z_{i}\right)}{2 \sigma_{R_{i}}^{2}}=\frac{\theta^{2}}{2 \sigma^{2}}=\frac{1}{2 \gamma}$. Thus, all $\lambda_{i}$ 's are equal to each other, and $\frac{R_{i}^{2}}{w^{2}\left(z_{i}\right)} \forall i$ are independent and identically distributed (i.i.d) exponential random variables. 


\section{A. First Upper Bound}

By substituting the expressions of $\mathcal{S}$ and $\beta_{m}$ in (59), we have that

$$
\begin{aligned}
\mathbb{P}(\mathcal{O}) & =\mathbb{P}\left(\left\{\frac{P_{t}^{2}}{\sigma_{n}^{2} \sum_{\ell=0}^{M-1} K_{\ell} \exp \left(\sum_{i=0}^{\ell}\left(\frac{R_{i}^{2}}{w^{2}\left(z_{i}\right)}+2 \alpha z_{i}\right)\right)} \leq \Upsilon_{\mathrm{th}}\right\}\right) \\
& =\mathbb{P}\left(\left\{\sum_{\ell=0}^{M-1} K_{\ell} \exp \left(\sum_{i=0}^{\ell}\left(\frac{R_{i}^{2}}{w^{2}\left(z_{i}\right)}+2 \alpha z_{i}\right)\right) \geq \frac{P_{t}^{2}}{\Upsilon_{\mathrm{th}} \sigma_{n}^{2}}\right\}\right)
\end{aligned}
$$

where $K_{\ell}:=\frac{4^{\ell}}{a^{4(\ell+1)}} \prod_{k=0}^{\ell-1} G_{k}^{-2} \prod_{j=0}^{\ell} w^{4}\left(z_{j}\right)$. Let us now define the weights $\mathcal{K}_{\ell}:=\frac{K_{\ell}}{\sum_{\ell=0}^{M-1} K_{\ell}}$. We know that $0 \leq \mathcal{K}_{\ell} \leq 1$ for all $\ell$. We then have that

$$
\mathbb{P}(\mathcal{O})=\mathbb{P}\left(\left\{\sum_{\ell=0}^{M-1} \mathcal{K}_{\ell} \exp \left(\sum_{i=0}^{\ell}\left(\frac{R_{i}^{2}}{w^{2}\left(z_{i}\right)}+2 \alpha z_{i}\right)\right) \geq \frac{P_{t}^{2}}{\Upsilon_{\mathrm{th}} \sigma_{n}^{2} \sum_{\ell=0}^{M-1} K_{\ell}}\right\}\right) .
$$

The outage probability in (63) is not straightforward to compute analytically. However, we may realize an upper bound on outage probability as:

$$
\begin{aligned}
\mathbb{P}(\mathcal{O}) & \leq \mathbb{P}\left(\left\{\sum_{\ell=0}^{M-1} \exp \left(\sum_{i=0}^{\ell}\left(\frac{R_{i}^{2}}{w^{2}\left(z_{i}\right)}+2 \alpha z_{i}\right)\right) \geq \frac{P_{t}^{2}}{\Upsilon_{\mathrm{th}} \sigma_{n}^{2} \sum_{\ell=0}^{M-1} K_{\ell}}\right\}\right) \\
& \leq \mathbb{P}\left(\left\{\sum_{\ell=0}^{M-1} \exp \left(\sum_{i=0}^{M-1}\left(\frac{R_{i}^{2}}{w^{2}\left(z_{i}\right)}+2 \alpha z_{i}\right)\right) \geq \frac{P_{t}^{2}}{\Upsilon_{\mathrm{th}} \sigma_{n}^{2} \sum_{\ell=0}^{M-1} K_{\ell}}\right\}\right) \\
& \leq \mathbb{P}\left(\left\{\sum_{i=0}^{M-1} \frac{R_{i}^{2}}{w^{2}\left(z_{i}\right)} \geq \ln \left(\frac{P_{t}^{2}}{M \Upsilon_{\mathrm{th}} \sigma_{n}^{2} \sum_{\ell=0}^{M-1} K_{\ell}}\right)-2 \alpha D\right\}\right) .
\end{aligned}
$$

As discussed before, the quantity $\frac{R_{k}^{2}}{w^{2}\left(z_{k}\right)}$ is an exponential random variable with common parameter $\lambda=\frac{1}{2 \gamma}$. Hence, we have that

$$
\mathbb{P}(\mathcal{O}) \leq \mathbb{P}_{u}(\mathcal{O}):=\mathbb{P}\left(\left\{W \geq \Upsilon_{\text {th }}^{\prime}\right\}\right)
$$

where $W:=\sum_{i=0}^{M-1} \frac{R_{i}^{2}}{w^{2}\left(z_{i}\right)}$ and

$$
\Upsilon_{\mathrm{th}}^{\prime}:=\ln \left(\frac{P_{t}^{2}}{\sigma_{n}^{2} \Upsilon_{\mathrm{th}} M \sum_{\ell=0}^{M-1} K_{\ell}}\right)-2 \alpha D
$$

For any positive integer $M$, we have that $W:=\sum_{k=0}^{M-1} \frac{R_{k}^{2}}{w^{2}\left(z_{k}\right)}$ is an $\operatorname{Erlang}(M, \lambda)$ random variable whose density function is given by

$$
f_{W}(x)=\frac{\lambda^{M} x^{M-1} e^{-\lambda x}}{(M-1) !}, \quad x>0
$$


Then, the upper bound on the outage probability is

$$
\begin{aligned}
\mathbb{P}_{u}(\mathcal{O}) & =\int_{\Upsilon_{\mathrm{th}}^{\prime}}^{\infty} f_{W}(x) d x=\sum_{n=0}^{M-1} \frac{1}{n !} e^{-\lambda \Upsilon_{\mathrm{th}}^{\prime}}\left(\lambda \Upsilon_{\mathrm{th}}^{\prime}\right)^{n} \cdot \mathbf{1}_{[0, \infty)}\left(\Upsilon_{\mathrm{th}}^{\prime}\right) \\
& =\exp \left(-\frac{\Upsilon_{\mathrm{th}}^{\prime}}{2 \gamma}\right) \sum_{n=0}^{M-1} \frac{1}{n !}\left(\frac{\Upsilon_{\mathrm{th}}^{\prime}}{2 \gamma}\right)^{n} \cdot \mathbf{1}_{[0, \infty)}\left(\Upsilon_{\mathrm{th}}^{\prime}\right)
\end{aligned}
$$

a) CSI-Assisted Relays: Finally, when $G_{i}=\frac{1}{\mathrm{~h}\left(z_{i}\right)}$, we have that

$$
K_{\ell}=\left(\frac{z_{\ell} \theta}{a}\right)^{4}\left(\frac{1}{1+\gamma}\right)^{2 \ell} \exp \left(-2 \alpha \sum_{k=0}^{\ell-1} z_{k}\right), \quad 0 \leq \ell \leq M-2,
$$

and this expression of $K_{\ell}$ is substituted in the threshold in (66) to compute the bound in (68).

\section{B. Second Upper Bound}

The $\beta_{\ell}$ in (58) is upper bounded by $\beta_{\ell}^{\prime}$ where

$$
\beta_{\ell}^{\prime}:=\frac{\left(\prod_{k=\ell}^{M-2} G_{k}\right)\left(\pi a^{2}\right)^{M-(\ell+1)} \exp \left(-\alpha \sum_{i=\ell+1}^{M-1} z_{i}\right)}{\prod_{j=\ell+1}^{M-1}\left(2 \pi w^{2}\left(z_{j}\right)\right)}
$$

Thus, $N=\sum_{\ell=0}^{M-1} N_{\ell} \beta_{\ell}^{\prime}$ and the variance of $N$ in this case is $\sigma_{N}^{2}=\sigma_{n}^{2} \sum_{\ell=0}^{M-1}\left(\beta_{\ell}^{\prime}\right)^{2}$. Thus,

$$
\mathbb{P}(\mathcal{O}) \leq \mathbb{P}_{u}(\mathcal{O}):=\mathbb{P}\left(\left\{\ln \left(\frac{\mathcal{S}^{2}}{\sigma_{n}^{2} \sum_{\ell=0}^{M-1}\left(\beta_{\ell}^{\prime}\right)^{2}}\right) \leq \ln \Upsilon_{\text {th }}\right\}\right)
$$

The second upper bound on the outage probability is given by

$$
\mathbb{P}_{u}(\mathcal{O})=\mathbb{P}\left(\left\{W \geq \Upsilon_{\text {th }}^{\prime}\right\}\right)
$$

where $W:=\sum_{i=0}^{M-1} \frac{R_{i}^{2}}{w^{2}\left(z_{i}\right)}$ is an $\operatorname{Erlang}(M, \lambda)$ random variable (as defined earlier) and

$$
\Upsilon_{\text {th }}^{\prime}:=\ln \left(\frac{P_{t}^{2}\left(a^{2}\right)^{2 M}\left(\prod_{k=0}^{M-2} G_{k}\right)^{2}}{2^{2(M-1)} \Upsilon_{\text {th }}\left(\prod_{j=0}^{M-1} w^{2}\left(z_{j}\right)\right)^{2} \sigma_{n}^{2} \sum_{\ell=0}^{M-1}\left(\beta_{\ell}^{\prime}\right)^{2}}\right)-2 \alpha D
$$

a) CSI-Assisted Relays: It can be shown that when $G_{i}=\frac{1}{\mathrm{~h}\left(z_{i}\right)}$ for $i=0,1, \ldots, M-1$, we have that

$$
\beta_{\ell}^{\prime}=\frac{z_{\ell}^{2}}{z_{M-1}^{2}} \exp \left(\alpha\left(z_{\ell}-z_{M-1}\right)\right)(1+\gamma)^{M-(\ell+1)}, \quad 0 \leq \ell \leq M-1
$$

and

$$
\Upsilon_{\mathrm{th}}^{\prime}=\ln \left(\frac{P_{t}^{2} a^{4}(1+\gamma)^{2(M-1)}}{\Upsilon_{\mathrm{th}} \theta^{4} \sigma_{n}^{2} \sum_{m=0}^{M-1} z_{m}^{4} e^{2 \alpha z_{m}}(1+\gamma)^{2(M-(m+1))}}\right) .
$$


The final expression of $\mathbb{P}_{u}(\mathcal{O})$ is the same as 68).

\section{Optimal Relay Positions}

We will later show (numerically) that the second upper bound furnishes a tighter bound as compared to the first upper bound. Therefore, in this section, our goal is to minimize the second upper bound as a function of $\boldsymbol{z}$.

In order to minimize the second upper bound, we note that the random variable $W$ in $(72)$ is not a function of $\boldsymbol{z}$. However, the threshold $\Upsilon_{\text {th }}^{\prime}$ given in $(75)$ is a function of $z$, and that the dependence of $\Upsilon_{\text {th }}^{\prime}$ on $\boldsymbol{z}$ is through the factor $\sum_{m=0}^{M-1} z_{m}^{4} e^{2 \alpha z_{m}}(1+\gamma)^{2(M-(m+1))}$. Therefore, we will minimize the outage probability bound by minimizing this factor with respect to $\boldsymbol{z}$. In this regard, we set $z_{m}=x_{m} D$ where $\sum_{m=0}^{M-1} x_{m}=1$ and $x_{m} \geq 0$ for all $m$. We now have the following (unconstrained) optimization problem on our hands:

1) Unconstrained Optimization

$$
\begin{array}{ll}
\underset{x_{0}, \ldots, x_{M-1}}{\operatorname{minimize}} & \sum_{m=0}^{M-1}\left(x_{m} D\right)^{4} e^{2 \alpha x_{m} D}(1+\gamma)^{2(M-(m+1))} \\
\text { subject to } & \text { i) } \sum_{m=0}^{M-1} x_{m}=1, \\
& \text { ii) } x_{m} \geq 0, \quad 0 \leq m \leq M-1 .
\end{array}
$$

As was the case with the optimization of probability of error, the objective function in (76) is a convex function of $\boldsymbol{x}$ with constraints that form convex sets. Thus, we first solve the first order Karush-Kuhn-Tucker conditions in order to reach a candidate solution for this convex optimization problem.

In order to find the solution, we follow the same line of reasoning as in Section $\mathrm{V}$-B where we optimized the probability of error. In order to save space, we are not discussing the intermediate steps here. The final result is discussed for two special scenarios: i) when $\gamma \approx 0$, and ii) when $\alpha \approx 0$.

a) $\gamma \approx 0:$ When $\gamma \approx 0$, the solution is the same as the solution of optimization problem (43) regarding the error probability. In this case, $x_{m}^{*}=\frac{1}{M}$ for all $m$.

b) $\alpha \approx 0$ : When $\alpha \approx 0$, we have that $x_{1}^{*}=(1+\gamma)^{\frac{2}{3}} x_{0}^{*}, x_{2}^{*}=(1+\gamma)^{\frac{4}{3}} x_{0}^{*}, \ldots, x_{M-1}^{*}=(1+\gamma)^{\frac{2(M-1)}{3}} x_{0}^{*}$, and from the last equation in the equation set (46), we have that

$$
x_{m}^{*}=\left(\frac{1-(1+\gamma)^{\frac{2}{3}}}{1-(1+\gamma)^{\frac{2 M}{3}}}\right)(1+\gamma)^{\frac{2 m}{3}}, \quad 0 \leq m \leq M-1 .
$$


We note that for this special case, we obtain ascending values of $x_{m}^{*}$ 's for the optimal scenario: $x_{0}^{*}<$ $x_{1}^{*}<\cdots<x_{M-1}^{*}$. Additionally, we note that all $x_{m}^{*}>0$.

It can also be easily shown that $\boldsymbol{x}^{*}:=\left[\begin{array}{llll}x_{0}^{*} & x_{1}^{*} & \cdots & x_{M-1}^{*}\end{array}\right]^{T}$ is a strict local minimizer by computing the Hessian matrix at $\boldsymbol{x}^{*}$.

2) Constrained Optimization of Relay Positions

a) Maximum Gain Constraint:

$$
\begin{array}{ll}
\underset{x_{0}, \ldots, x_{M-1}}{\operatorname{minimize}} & \sum_{m=0}^{M-1}\left(x_{m} D\right)^{4} e^{2 \alpha x_{m} D}(1+\gamma)^{2(M-(m+1))} \\
\text { subject to } & \text { i) } \sum_{m=0}^{M-1} x_{m}=1, \\
& \text { ii) } x_{m} \geq 0, \quad 0 \leq m \leq M-1, \\
& \text { iii) } D^{2} x_{m}^{2} e^{\alpha x_{m} D} \leq \frac{\mathcal{G}_{u} a^{2}}{2 \theta^{2}(1+\gamma)}, \quad 0 \leq m \leq M-2 .
\end{array}
$$

For the gain constraint, there is only one point that lies in $B$, which is the optimal point. This optimal point satisfies $x_{0}^{*}=x_{1}^{*}=\cdots=x_{M-2}^{*}$ and $x_{M-1}^{*}=1-\sum_{m=0}^{M-2} x_{m}^{*}$.

When $\gamma \approx 0$, this problem reduces to the optimization problem in $(35)$. For the case when $\alpha \approx 0$, we note from (77) that the gain constraint is active when $\mathcal{G}_{u} \leq \frac{2 D^{2} \theta^{2}(1+\gamma)}{a^{2}}\left(x_{M-2}^{*}\right)^{2}$, where $x_{M-2}^{*}:=\left(\frac{1-(1+\gamma)^{\frac{2}{3}}}{1-(1+\gamma)^{\frac{2 M}{3}}}\right)(1+\gamma)^{\frac{2(M-2)}{3}}$. When the constraint is active, the optimal point is $x_{m}^{*}=\sqrt{\frac{\mathcal{G}_{u} a^{2}}{2 D^{2} \theta^{2}(1+\gamma)}}$ for $0 \leq m \leq M-2$, and $x_{M-1}^{*}=1-\sum_{m=0}^{M-2} x_{m}^{*}$.

b) Maximum Power Constraint:

$$
\begin{array}{ll}
\underset{x_{0}, \ldots, x_{M-1}}{\operatorname{minimize}} & \sum_{m=0}^{M-1}\left(x_{m} D\right)^{4} e^{2 \alpha x_{m} D}(1+\gamma)^{2(M-(m+1))} \\
\text { subject to } & \text { i) } \sum_{m=0}^{M-1} x_{m}=1, \\
& \text { ii) } x_{m} \geq 0, \quad 0 \leq m \leq M-1, \\
& \text { iii })\left(\sum_{m=0}^{M-2} D^{4} x_{m}^{4} e^{2 \alpha x_{m} D}(1+\gamma)^{2(M-(m+1))}\right)^{\frac{1}{2}} \leq \frac{a^{2}\left(\mathcal{P}_{u}-2 P_{t}(1+\gamma)^{M-1}\right)}{2 \theta^{2} N^{\star}} .
\end{array}
$$

When $\gamma \approx 0$, this problem reduces to the optimization problem in (37).

Regarding $\gamma>>0$, as with the gain constraint, the optimal point lies in the boundary set $B$. However, $B$ may contain more than one point that satisfy the maximum power constraint. If the power constraint 
in (79) is active, then

$$
\left(\sum_{m=0}^{M-2} D^{4}\left(x_{m}^{*}\right)^{4} e^{2 \alpha x_{m}^{*} D}(1+\gamma)^{2(M-(m+1))}\right)^{\frac{1}{2}}>\frac{a^{2}\left(\mathcal{P}_{u}-2 P_{t}(1+\gamma)^{M-1}\right)}{2 \theta^{2} N^{\star}}
$$

where $x_{m}^{*}$ is furnished by (77). This means that there exists a $\frac{D_{0}}{\mathcal{D}}<D$ such that

$$
\left(\sum_{m=0}^{M-2}\left(\frac{D_{0}}{\mathcal{D}}\right)^{4}\left(x_{m}^{*}\right)^{4} e^{2 \alpha x_{m}^{*} \frac{D_{0}}{\mathcal{D}}}(1+\gamma)^{2(M-(m+1))}\right)^{\frac{1}{2}}=\frac{a^{2}\left(\mathcal{P}_{u}-2 P_{t}(1+\gamma)^{M-1}\right)}{2 \theta^{2} N^{\star}}
$$

where $\mathcal{D}:=\sum_{m=0}^{M-2} x_{m}^{*}$ and $\mathcal{D}<1$. For $\alpha \approx 0$, we now claim that the vector

$$
\tilde{\boldsymbol{z}}_{B}:=\left[\begin{array}{lllll}
\frac{D_{0}}{\mathcal{D}} x_{0}^{*} & \frac{D_{0}}{\mathcal{D}} x_{1}^{*} & \cdots & \frac{D_{0}}{\mathcal{D}} x_{M-2}^{*} & D-D_{0}
\end{array}\right]^{T}
$$

is the optimal hop distance vector that will minimize the outage probability bound under the power constraint. We reiterate that $\tilde{\boldsymbol{z}}_{B} \in B$, and the sum of the first $M-1$ elements of $\tilde{\boldsymbol{z}}_{B}$ is $D_{0}$.

c) Proof by Contradiction: Let us assume that another vector

$$
\boldsymbol{z}_{B}^{\prime}:=\left[\begin{array}{lllll}
\frac{D_{0}^{\prime}}{\mathcal{D}} x_{0}^{\prime} & \frac{D_{0}^{\prime}}{\mathcal{D}} x_{1}^{\prime} & \cdots & \frac{D_{0}^{\prime}}{\mathcal{D}} x_{M-2} & D-D_{0}^{\prime}
\end{array}\right]^{T}
$$

which lies in $B$, and $x_{m}^{\prime}>0$ for $0 \leq m \leq M-2$ and $\sum_{m=0}^{M-2} x_{m}^{\prime}=\mathcal{D}$. We assume that $\boldsymbol{z}_{B}$ is the optimal hop distance vector and that $\boldsymbol{z}_{B}^{\prime} \neq \tilde{\boldsymbol{z}}_{B}$.

If $\boldsymbol{z}_{B}^{\prime}$ is in $B$, then $D_{0}^{\prime}<D_{0}$ because both $\tilde{\boldsymbol{z}}_{B}$ and $\boldsymbol{z}_{B}^{\prime}$ being boundary points implies

$$
\left(\sum_{m=0}^{M-2}\left(\frac{D_{0}}{\mathcal{D}}\right)^{4}\left(x_{m}^{*}\right)^{4} e^{2 \alpha x_{m}^{*} \frac{D_{0}}{\mathcal{D}}}(1+\gamma)^{2(M-(m+1))}\right)^{\frac{1}{2}}=\left(\sum_{m=0}^{M-2}\left(\frac{D_{0}^{\prime}}{\mathcal{D}}\right)^{4}\left(x_{m}^{\prime}\right)^{4} e^{2 \alpha x_{m}^{\prime} \frac{D_{0}^{\prime}}{\mathcal{D}}}(1+\gamma)^{2(M-(m+1))}\right)^{\frac{1}{2}}
$$

and since $x_{m}^{*}$ for $0 \leq m \leq M-2$ minimize $\left(\sum_{m=0}^{M-2} \mathcal{K}^{4}\left(x_{m}^{*}\right)^{4} e^{2 \alpha x_{m}^{*} \mathcal{K}}(1+\gamma)^{2(M-(m+1))}\right)^{\frac{1}{2}}$ for any arbitrary positive number $\mathcal{K}, D_{0}^{\prime}$ has to be less than $D_{0}$ in order for the equality in (84) to hold.

Let us consider the hop distance vector $\tilde{\boldsymbol{z}}^{\prime}:=\left[\begin{array}{lllll}\frac{D_{0}^{\prime}}{\mathcal{D}} x_{0}^{*} & \frac{D_{0}^{\prime}}{\mathcal{D}} x_{1}^{*} & \cdots & \frac{D_{0}^{\prime}}{\mathcal{D}} x_{M-2}^{*} & D-D_{0}^{\prime}\end{array}\right]^{T}$. The sum of first $M-1$ terms of this vector is equal to $D_{0}^{\prime}$. This vector $\tilde{z}$ will produce a smaller value of the term $\left(\sum_{m=0}^{M-2} \frac{D_{0}^{\prime}}{\mathcal{D}}\left(x_{m}\right)^{4} e^{2 \alpha x_{m}} \frac{D_{0}^{\prime}}{\mathcal{D}}\right)^{\frac{1}{2}}$ compared to $\boldsymbol{z}_{B}^{\prime}$. Hence, this point satisfies the power constraint (even though the constraint is satisfied with a strict inequality which implies that $\tilde{\boldsymbol{z}}^{\prime} \notin B$ ). Moreover, we have that,

$$
\left(\sum_{m=0}^{M-2}\left(\frac{D_{0}^{\prime}}{\mathcal{D}}\right)^{4}\left(x_{m}^{*}\right)^{4} e^{2 \alpha x_{m}^{*} \frac{D_{0}^{\prime}}{\mathcal{D}}}\right)+\left(D-D_{0}^{\prime}\right)^{4} e^{2 \alpha\left(D-D_{0}^{\prime}\right)}<\left(\sum_{m=0}^{M-2}\left(\frac{D_{0}^{\prime}}{\mathcal{D}}\right)^{4}\left(x_{m}^{\prime}\right)^{4} e^{2 \alpha x_{m}^{\prime}} \frac{D_{0}^{\prime}}{\mathcal{D}}\right)+\left(D-D_{0}^{\prime}\right)^{4} e^{2 \alpha\left(D-D_{0}^{\prime}\right)}
$$


where the quantity on the left of the inequality is the objective function corresponding to $\tilde{\boldsymbol{z}}^{\prime}$, and the quantity on the right of the inequality is the objective function corresponding to $\boldsymbol{z}_{B}^{\prime}$. Thus, $\mathbb{P}\left(\tilde{\boldsymbol{z}}^{\prime}\right) \leq \mathbb{P}\left(\boldsymbol{z}_{B}^{\prime}\right)$. Additionally, $\mathbb{P}\left(\tilde{\boldsymbol{z}}_{B}\right) \leq \mathbb{P}\left(\tilde{\boldsymbol{z}}^{\prime}\right)$ since $\tilde{\boldsymbol{z}}_{B} \in B$ and $\tilde{\boldsymbol{z}}^{\prime} \notin B$. Thus, we have that $\mathbb{P}\left(\tilde{\boldsymbol{z}}_{B}\right) \leq \mathbb{P}\left(\boldsymbol{z}_{B}^{\prime}\right)$, which is a contradiction. Hence, it follows that $\boldsymbol{z}_{B}^{*}=\tilde{\boldsymbol{z}}_{B}$.

\section{Simulation Results}

In this section, we plot the error and outage probability bound curves for different optimization scenarios discussed in Section $\mathrm{V}$ and Section VI. Here, we use the term "unoptimized" system to describe the equidistant relay positioning scheme where the distance between any two relays in the backhaul are equal to each other.

For the purpose of simulations, the system parameters have been selected after a careful search of literature on state-of-the-art FSO systems. Important references that we looked at for the selection of suitable parameter values are [10], [5], [21], [22], [23], [24]. The selected parameter values are as follows: Total end-to-end link distance $D=10000 \mathrm{~m}$, the maximum allowable transmitted power limit $\mathcal{P}_{u}: 50-80$ $\mathrm{W}$, the power at the first relay in the backhaul $P_{t}: 2-20 \mathrm{~W}$, receiver noise standard deviation $\sigma_{n}: 0.1-60$ $\mu \mathrm{W}$, beamwidth angle-of-arrival standard deviation $\sigma: 10-50 \mathrm{mrad}$ and the upper gain limit of relay $\mathcal{G}_{u}: 50-70 \mathrm{~dB}$. The optical wavelength is $1550 \mathrm{~nm}$ and the beam waist $w_{0}=10-50 \mu \mathrm{m}$, which leads to a beamwidth $\theta$ of 5-50 mrad. The value of attenuation coefficient $\alpha$ due to fog or dirt is $1-5 \times 10^{-4}$ which is a dimensionless parameter.

\section{A. Minimization of Error Probability}

Fig. 3 shows the probability of error as a function of angle-of-arrival standard deviation for the unconstrained optimization (left) and the optimization under gain constraint. We note that the performance gap between the optimized and unoptimized schemes gets wider when the gain constraint is implemented.

Fig. 4 depicts probability of error as a function of beamwidth $\theta$ for the optimized and unoptimized scenarios under condition of no optimization constraint. As $\theta$ becomes large and $\gamma$ becomes small, the optimized scheme converges to the equidistant or unoptimized scheme (please see Equation (47)). Thus, we see the gap between optimized and unoptimized schemes close as $\theta$ becomes large.

Fig. 5illustrates the probability of error as a function of noise standard deviation $\sigma_{n}$ for the optimization performance under gain and power constraints. Fig. 6 considers the error probability as a function of 

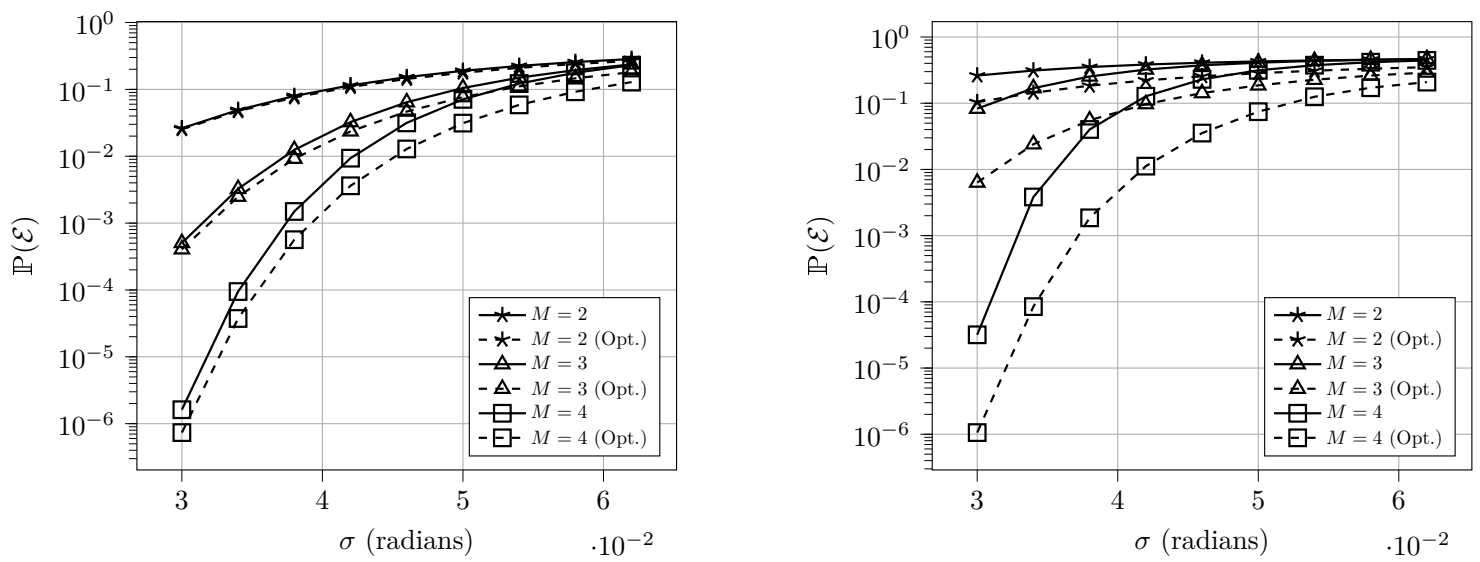

Fig. 3. The figure shows the average bit error rate plots as a function of angle-of-arrival standard deviation for different number of relays $M$ in the backhaul. The two subfigures compare the performance of the optimized and unoptimized schemes under the no constraint condition (left), and when the system is constrained by a finite gain $\mathcal{G}_{u}$ on each drone (right). The parameter values (for both the figures) are as follows: Link distance $D=10000$ meters, transmitted power $P_{t}=20$ Watts, beamwidth $\theta=0.03$ radians, aperture radius $a=0.2 \mathrm{~m}$, noise standard deviation $\sigma_{n}=3 \mu \mathrm{V}$, the attenuation coefficient $\alpha=0$, and the gain constraint $\mathcal{G}_{u}=5 \times 10^{5}$ for the figure on the right.

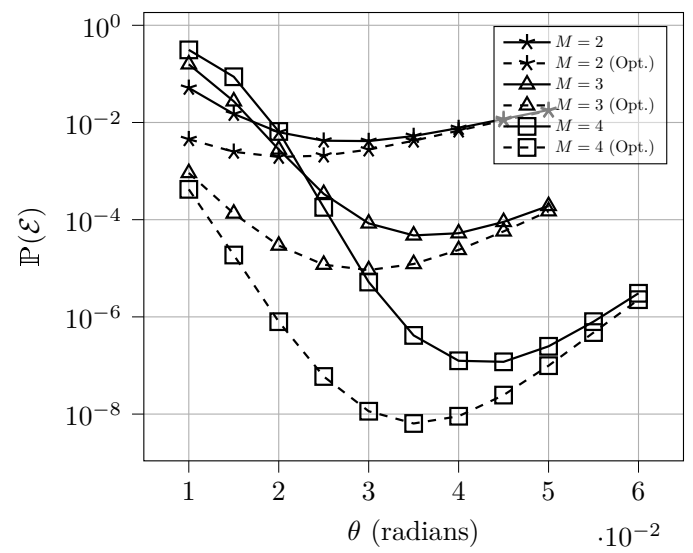

Fig. 4. This figure shows the average bit error rate as a function of beamwidth $\theta$ for the optimized and unoptimized schemes for unconstrained optimization. The common parameter values for the two figures are as follows: Link distance $D=10000$ meters, transmitted power $P_{t}=20$ Watts, angle-of-arrival standard deviation $\sigma=0.02$ radians, aperture radius $a=0.2 \mathrm{~m}$, and the noise standard deviation $\sigma_{n}=1 \mu \mathrm{V}$. For the figure on the right, the upper gain limit is $\mathcal{G}_{u}=61.76 \mathrm{~dB}$. The attenuation coefficient $\alpha=0$ for both the figures.

gain constraint $\mathcal{G}_{u}$. We note that as the value of $\mathcal{G}_{u}$ becomes large enough, the gain constraint becomes inactive. At this point, any further improvement in gain does not change or improve the error probability performance.

Fig. 7] shows the error probability as a function of number of hops $M$ for favorable channel conditions or small $\gamma(\gamma=0.027)$ when the total average power of the system is constant. As discussed in Section V-C1. the probability of error diminishes with an increase in the number of relays in the backhaul. The subfigure on the right in the same figure shows the performance comparison for optimized and unoptimized schemes 


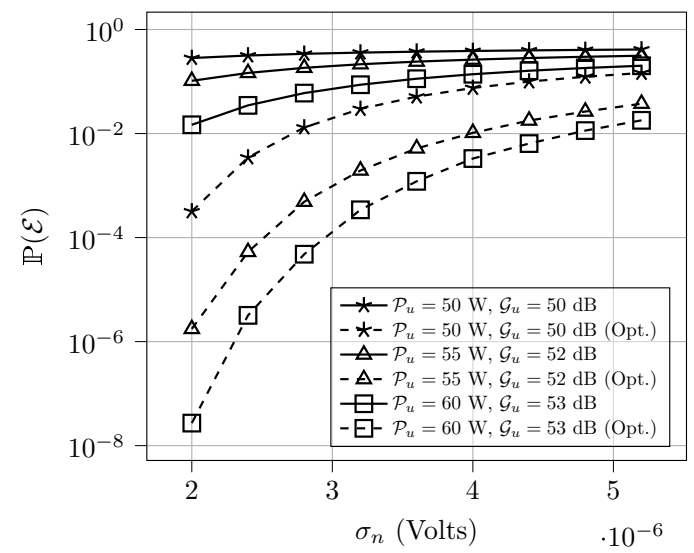

Fig. 5. This figure shows the average bit error rate as a function of noise standard deviation $\sigma_{n}$ under the conditions of finite maximum power and gain constraints for the optimized and unoptimized schemes. The parameter values are as follows: Link distance $D=10000$ meters, transmitted power $P_{t}=20$ Watts, angle-of-arrival standard deviation $\sigma=0.01$ radians, beamwidth $\theta=0.03$ radians, aperture radius $a=0.2 \mathrm{~m}$, and the attenuation coefficient $\alpha=10^{-4}$.

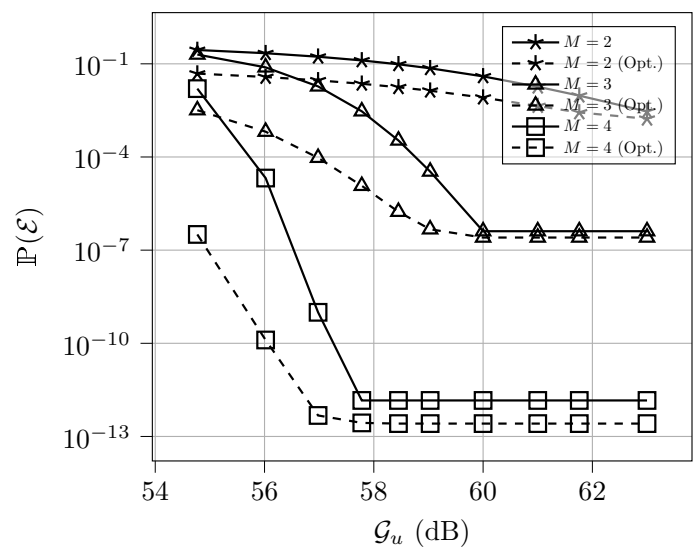

Fig. 6. This figure shows the average bit error rate as a function of upper gain limit $\mathcal{G}_{u}$. The parameter values are as follows: Link distance $D=10000$ meters, transmitted power $P_{t}=20$ Watts, angle-of-arrival standard deviation $\sigma=0.03$ radians, beamwidth $\theta=0.03$ radians, aperture radius $a=0.2 \mathrm{~m}$, The noise standard deviation $\sigma_{n}=2 \mu \mathrm{V}$. The value of attenuation coefficient $\alpha=0$.

under the gain constraint. Since the gain constraint will become inactive at a large enough $M$ for the unoptimized or equidistant scheme (please see Equation (27)), the two curves will meet at a certain value of $M$ as depicted in the figure. In contrast to Fig. 7, Fig. 8 shows the error probability for large $\gamma$ $(\gamma=9)$ which corresponds to a large pointing error or poor channel conditions. Here, as discussed in Section $\mathrm{V}-\mathrm{C} 2$, the error probability increases with an increase in the number of relays in the backhaul under total average power constraint. 

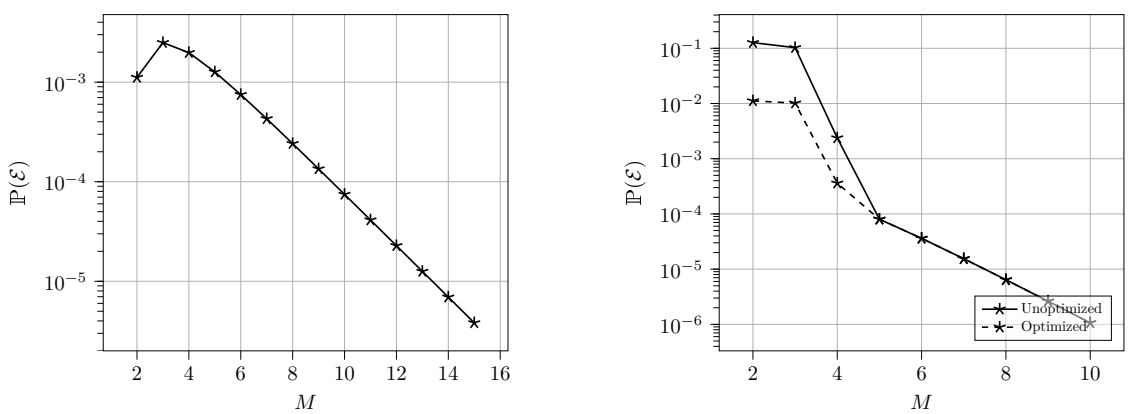

Fig. 7. This figure shows the average bit error rate as a function of number of hops $M$ for a constant total average power for a low $\gamma$ scenario. The figure on left corresponds to equidistant scheme (no constraints) and the figure on right shows the comparison between the equidistant and optimized schemes for a gain constraint of $\mathcal{G}_{u}=2.5 \times 10^{5}$. Other parameter values are as follows: Link distance $D=10000$ meters, total average transmitted power $\mathcal{P}_{a}=100$ Watts, angle-of-arrival standard deviation $\sigma=0.005$ radians, beamwidth $\theta=0.03$ radians, aperture radius $a=0.2$ meters, and the attenuation coefficient $\alpha=0$. The noise standard deviation is $\sigma_{n}=10 \mu \mathrm{V}$ (left) and $\sigma_{n}=8 \mu \mathrm{V}$ (right).
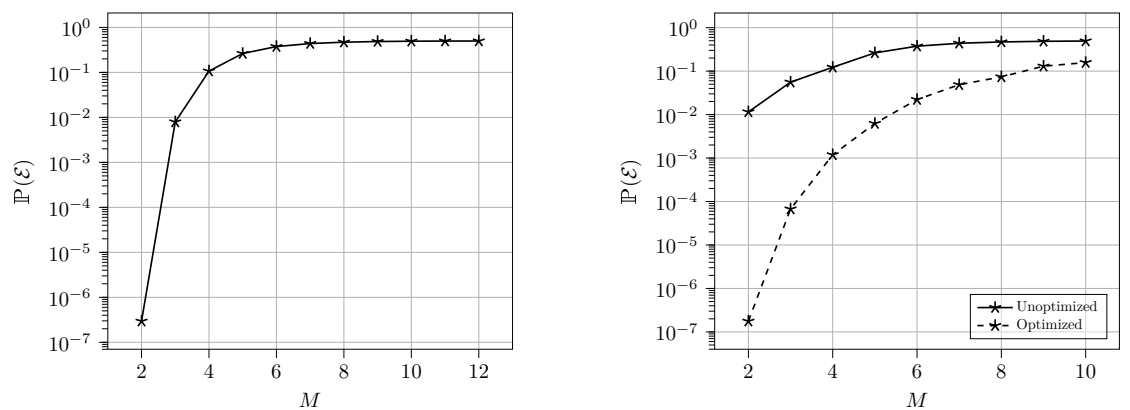

Fig. 8. This figure shows the average bit error rate as a function of number of hops $M$ for a constant total average power for a high $\gamma$ scenario. The figure on left corresponds to equidistant scheme (no constraints) and the figure on right shows the comparison between the equidistant and optimized schemes for a gain constraint of $\mathcal{G}_{u}=1 \times 10^{6}$. Other parameter values are as follows: Link distance $D=10000$ meters, total average transmitted power $\mathcal{P}_{a}=100$ Watts, angle-of-arrival standard deviation $\sigma=0.06$ radians, beamwidth $\theta=0.02$ radians, aperture radius $a=0.2$ meters, and the attenuation coefficient $\alpha=0$. The noise standard deviation is $\sigma_{n}=8 \mu \mathrm{V}$.

\section{B. Minimization of Outage Probability}

Fig. 9 shows the comparison between the outage probability and its two upper bounds as a function of beamwidth $\theta$. We notice that upper bound 2 is tighter than upper bound 1 . Therefore, we have chosen upper bound 2 as our performance metric in the rest of the experimental work in this section.

Fig. 10 illustrates the outage probability bound as a function of the angle-of-arrival standard deviation $\sigma$ for the optimized versus unoptimized systems for constrained optimization. Fig. 11 shows the outage probability bound as a function of beamwidth $\theta$ for the unconstrained and constrained optimization scenario (only gain constraint is considered for constrained optimization).

Fig. 12 represents the outage probability bound as a function of upper gain limit $\mathcal{G}_{u}$. Since $\gamma$ is small in this case, $x_{m}^{*} \approx \frac{1}{M}$ for the unconstrained optimization (the reader may revisit the $\gamma \approx 0$ case in Section VI-C1). Therefore, we see that as the upper gain limit $\mathcal{G}_{u}$ approaches a large value and the gain 


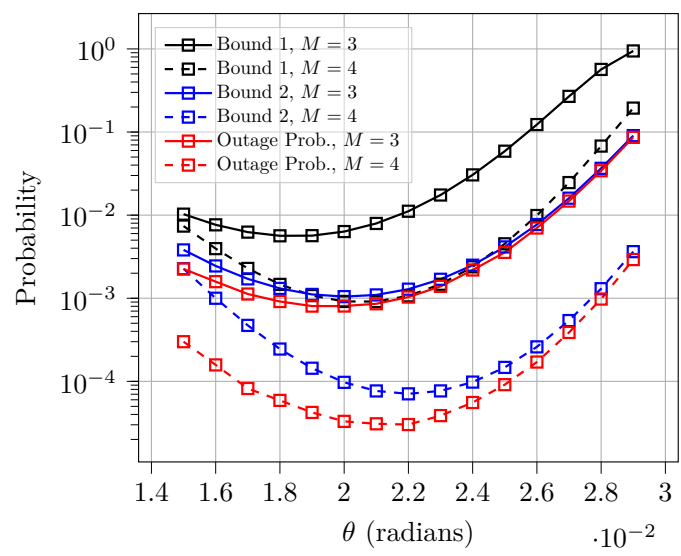

Fig. 9. This figure shows the outage probability and the two upper bounds as a function for beamwidth $\theta$. Other parameter values are as follows: Link distance $D=10000$ meters, transmitted power $P_{t}=20$ Watts, angle-of-arrival standard deviation $\sigma=0.01$ radians, aperture radius $a=0.2$ meters, attenuation coefficient $\alpha=10^{-4}$, upper limit on maximum power $\mathcal{P}_{u}=50$ Watts, threshold $\Upsilon_{\text {th }}=1$, and the noise standard deviation is $\sigma_{n}=2 \times 10^{-6}$ Volts.

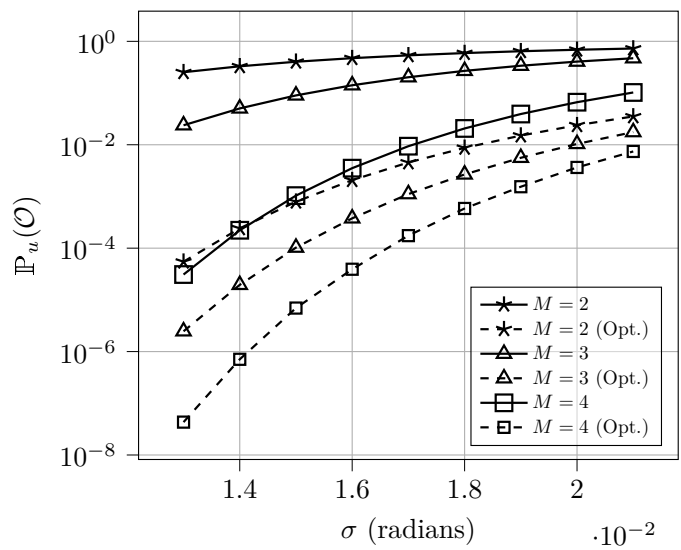

Fig. 10. This figure depicts the outage probability as a function of angle-of-arrival standard deviation $\sigma$. Other parameter values are as follows: Link distance $D=10000$ meters, transmitted power $P_{t}=20$ Watts, beamwidth $\theta=0.04$ radians, aperture radius $a=0.2 \mathrm{~m}$, attenuation coefficient $\alpha=10^{-4}$, upper limit on maximum power $\mathcal{P}_{u}=100$ Watts, the upper limit on gain $\mathcal{G}_{u}=3 \times 10^{5}$, threshold $\Upsilon_{\text {th }}=5$, and the noise standard deviation is $\sigma_{n}=1 \times 10^{-6}$ Volts.

constraint becomes inactive, the outage probability performances of optimized and unoptimized schemes become similar.

Finally, we examine the effect of number of hops $M$ on the outage probability performance when the total average power is held constant. Here, the outage probability bound is minimized only for a particular value of $M_{0}$, and for any other value of $M \neq M_{0}$, the bound is larger. This behavior of the outage probability bound as a function of $M$ is illustrated in Fig. 13 for the optimized and unoptimized schemes under gain constraints. 

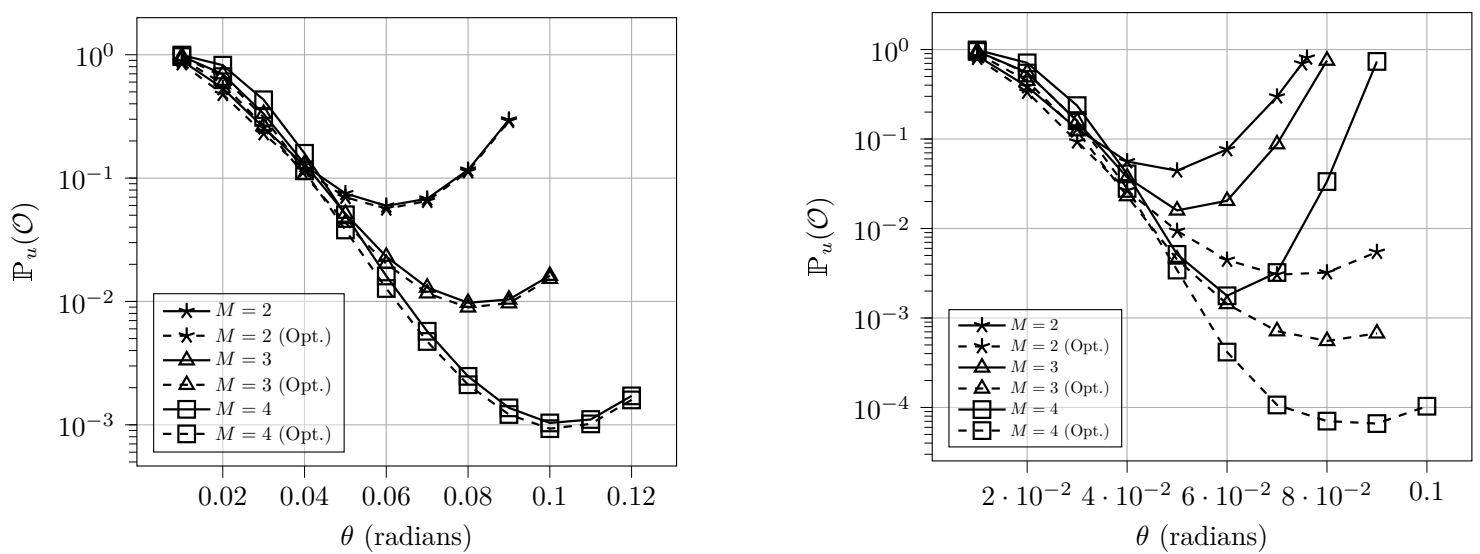

Fig. 11. This figure shows the outage probability as a function of beamwidth $\theta$ for the unconstrained optimization (left) and optimization with gain constraint (right). Other parameter values are as follows: Link distance $D=10000$ meters, transmitted power $P_{t}=20$ Watts, angle-of-arrival standard deviation $\sigma=0.03$ radians, aperture radius $a=0.2 \mathrm{~m}$, attenuation coefficient $\alpha=0$, the upper limit on gain $\mathcal{G}_{u}=1 \times 10^{6}$ (right figure), and the noise standard deviation is $\sigma_{n}=1 \times 10^{-6}$ Volts.

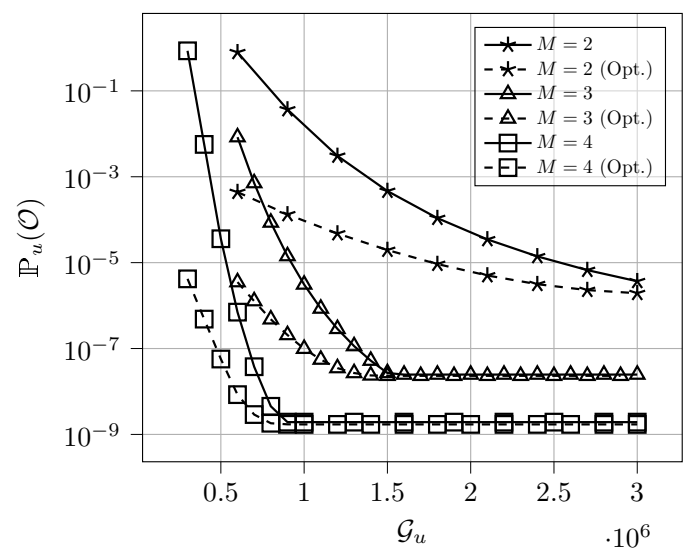

Fig. 12. This figure demonstrates the outage probability as a function of upper limit on the gain $\mathcal{G}_{u}$. Other parameter values are as follows: Link distance $D=10000$ meters, transmitted power $P_{t}=20$ Watts, angle-of-arrival standard deviation $\sigma=0.015$ radians, beamwidth $\theta=0.05$ radians, aperture radius $a=0.2 \mathrm{~m}$, attenuation coefficient $\alpha=0$, upper limit on maximum power $\mathcal{P}_{u}=100$ Watts, threshold $\Upsilon_{\text {th }}=5$, and the noise standard deviation is $\sigma_{n}=1 \times 10^{-6}$ Volts.

\section{ConcLusions}

In this study, we have shown that the hop distance parameter $z$ (or analogously, the relay positions along a serial relay link) in a free-space optical backhaul is an important parameter that can be exploited in order to minimize the end-to-end error and outage probabilities. When the gain and power constraints are not active, we showed analytically that the optimum hop distance vector is a strictly increasing sequence whose values depend on the factor $\gamma$ for both the error probability and outage probability bound. A larger $\gamma$ implies a larger ratio $\frac{z_{m+1}^{*}}{z_{m}^{*}}$ for $0 \leq m \leq M-2$ where $z_{m}^{*}$ is the optimal value of $z_{m}$.

When the gain constraint is active, the optimal solution for error and outage probabilities is a quasiequidis- 


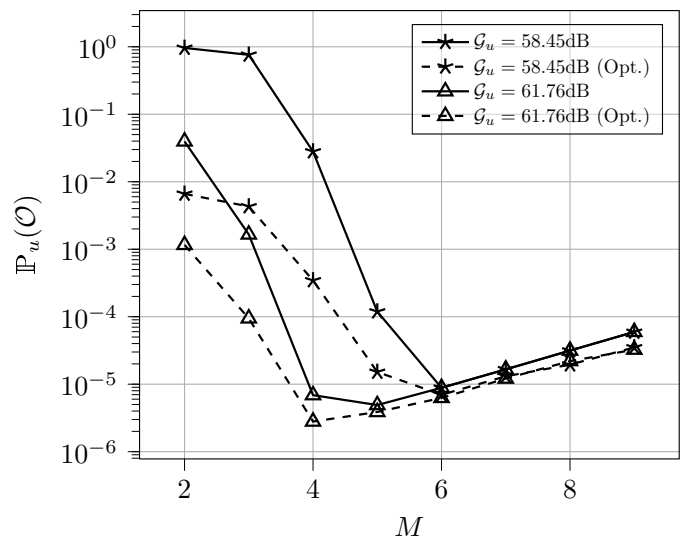

Fig. 13. This plot shows the outage probability as a function of number of total hops $M$. Other parameter values are as follows: Link distance $D=10000$ meters, the total average transmitted power $\mathcal{P}_{a}=200$ Watts, pointing error standard deviation $\sigma=0.02$ radians, beamwidth $\theta=0.05$ radians, aperture radius $a=0.2 \mathrm{~m}$, threshold $\Upsilon_{\mathrm{th}}=5$, attenuation coefficient $\alpha=2.5 \times 10^{-4}$, and the noise standard deviation is $\sigma_{n}=5 \times 10^{-7}$ Watts.

tant vector which is define in Section V-A3, Moreover, when the maximum power constraint is active for the special case of $\gamma \approx 0$, the optimal solution is once again a quasiequidistant vector. However, when $\gamma>>0$, the optimal hop distance vector for active power constraint is a strictly increasing sequence whose value depends on $\gamma$.

From the perspective of a constant total average power, a large number of low power relays in the backhaul is a better choice from the error probability perspective when channel conditions are relatively good (small $\gamma$ ). Conversely, for adverse channel conditions (large $\gamma$ ), a small number of high power relays minimizes the error probability. With regards to outage probability, only a certain number of relays will minimize the outage probability bound for a fixed total average power.

\section{REFERENCES}

[1] M. Alzenad, M. Z. Shakir, H. Yanikomeroglu, and M.-S. Alouini, "Fso-based vertical backhaul/fronthaul framework for 5g+ wireless networks," IEEE Communications Magazine, vol. 56, no. 1, pp. 218-224, 2018.

[2] M. S. Bashir and M.-S. Alouini, "Optimal power allocation between beam tracking and symbol detection channels in a free-space optical communications receiver,” Dec 2020. [Online]. Available:https://www.techrxiv.org/articles/preprint/Optimal_Power_Allocation_ Between_Beam_Tracking_and_Symbol_Detection_Channels_in_a_Free-Space_Optical_Communications_Receiver/13296563/1

[3] M. S. Bashir, M.-C. Tsai, and M.-S. Alouini, "Cramér-rao bounds for beam tracking with photon counting detector arrays in free-space optical communications," IEEE Open Journal of the Communications Society, vol. 2, pp. 1065-1081, 2021.

[4] M. S. Bashir and M. S. Alouini, "Free-space optical MISO communications with an array of detectors," IEEE Open Journal of the Communications Society, vol. 1, pp. 1765-1780, 2020.

[5] V. V. Mai and H. Kim, "Adaptive beam control techniques for airborne free-space optical communication systems," Applied Optics, vol. 57, no. 26, September 2018. 
[6] H. Al-Quwaiee, H. C. Yang, and M. -S. Alouini, "On the asymptotic capacity of dual-aperture FSO systems with a generalized pointing error model," IEEE Transactions on Wireless Communications, vol. 15, no. 9, pp. 6502-6512, September 2016.

[7] H. AlQuwaiee, H.-C. Yang, and M.-S. Alouini, "On the asymptotic capacity of dual-aperture FSO systems with generalized pointing error model," IEEE Trans. Wireless Commun., vol. 15, no. 9, pp. 6502-6512, 2016.

[8] C. B. Issaid, K.-H. Park, and M.-S. Alouini, "A generic simulation approach for the fast and accurate estimation of the outage probability of single hop and multihop FSO links subject to generalized pointing errors," IEEE Trans. Wireless Commun., vol. 16, no. 10, pp. 6822-6837, 2017.

[9] M. T. Dabiri, S. M. S. Sadough, and M. A. Khalighi, "Channel modeling and parameter optimization for hovering uav-based free-space optical links," IEEE Journal on Selected Areas in Communications, vol. 36, no. 9, pp. 2104-2113, 2018.

[10] A. A. Farid and S. Hranilovic, "Outage capacity optimization for free-space optical links with pointing errors," Journal of Lightwave Technology, vol. 25, no. 7, July 2007.

[11] Y. Ma, J.-Y. Wang, J.-B. Wang, M. Lin, H. Zhang, and C. Chang, "Outage performance analysis and parameter optimization of hovering uav-based fso system," in ICC 2020 - 2020 IEEE International Conference on Communications (ICC), 2020, pp. 1-6.

[12] V. V. Mai and H. Kim, "Beam size optimization and adaptation for high-altitude airborne free-space optical communication systems," IEEE Photonics Journal, vol. 11, no. 2, pp. 1-13, 2019.

[13] E. Zedini and M.-S. Alouini, "Multihop relaying over im/dd fso systems with pointing errors," J. Lightw. Technol., vol. 33, no. 23, pp. $5007-5015,2015$.

[14] M. A. Kashani, M. Safari, and M. Uysal, "Optimal relay placement and diversity analysis of relay-assisted free-space optical communication systems," IEEE/OSA Journal of Optical Communications and Networking, vol. 5, no. 1, pp. 37-47, 2013.

[15] M. Safari and M. Uysal, "Relay-assisted free-space optical communication," IEEE Transactions on Wireless Communications, vol. 7, no. 12, pp. 5441-5449, 2008.

[16] B. Zhu, J. Cheng, and L. Wu, "Optimal fso relay nodes placement with link obstacles and infeasible regions," in 2014 IEEE Global Communications Conference, 2014, pp. 2137-2142.

[17] H. G. Sandalidis, "Performance analysis of a laser ground-station-to-satellite link with modulated gamma-distributed irradiance fluctuations," J. Opt. Commun. Netw., vol. 2, no. 11, pp. 938-943, Nov 2010. [Online]. Available: http: //jocn.osa.org/abstract.cfm?URI=jocn-2-11-938

[18] M. A. Cox, L. Gailele, L. Cheng, and A. Forbes, "Modelling the memory of turbulence-induced beam wander," 2019.

[19] E. Zedini, H. Soury, and M.-S. Alouini, "On the performance analysis of dual-hop mixed FSO/RF systems," IEEE Trans. Wireless Commun., vol. 15, no. 5, pp. 3679-3689, 2016.

[20] M. S. Bashir and M.-S. Alouini, "Adaptive acquisition schemes for photon-limited free-space optical communications," IEEE Transactions on Communications, vol. 69, no. 1, pp. 416-428, 2021.

[21] A. Trichili, M. A. Cox, B. S. Ooi, and M.-S. Alouini, "Roadmap to free space optics," J. Opt. Soc. Am. B, vol. 37, no. 11, pp. A184-A201, Nov 2020. [Online]. Available: http://josab.osa.org/abstract.cfm?URI=josab-37-11-A184

[22] A. Trichili, K.-H. Park, M. Zghal, B. S. Ooi, and M.-S. Alouin, "Communicating using spatial mode multiplexing: Potentials, challenges and perspectives," IEEE Commun. Surveys Tuts., 2018.

[23] M. S. Bashir and M. R. Bell, "Optical beam position estimation in free-space optical communication," IEEE Transactions on Aerospace and Electronic Systems, vol. 52, no. 6, December 2016.

[24] — - "Optical beam position tracking in free-space optical communication systems," IEEE Transactions on Aerospace and Electronic Systems, vol. 20, no. 2, April 2018. 